\title{
Planning Natural Repointing Manoeuvres for Nano-Spacecraft
}

\author{
Craig Maclean Daniele Pagnozzi James D. Biggs \\ Advanced Space Concepts Laboratory, Department of Mechanical \& Aerospace Engineering, University of \\ Strathclyde, Glasgow, G1 IXJ, UK
}

\begin{abstract}
In this paper the natural dynamics of a rigid body are exploited to plan attitude manoeuvres for a small spacecraft. By utilising the analytical solutions of the angular velocities and making use of Lax pair integration, the time evolution of the attitude of the spacecraft in a convenient quaternion form is derived. This enables repointing manoeuvres to be generated by optimising the free parameters of the analytical expressions, the initial angular velocities of the spacecraft, to match prescribed boundary conditions on the final attitude of the spacecraft. This produces reference motions which can be tracked using a simple proportional-derivative controller. The natural motions are compared in simulation to a conventional quaternion feedback controller and found to require lower accumulated torque. A simple obstacle avoidance algorithm, exploiting the analytic form of natural motions, is also described and implemented in simulation. The computational efficiency of the motion planning method is discussed.
\end{abstract}

Keywords. Geometric mechanics, motion planning, nanospacecraft.

\begin{tabular}{|c|c|}
\hline$A_{1}, A_{2}, A_{3}$ & $\begin{array}{l}\text { NOMENCLATURE } \\
\text { Basis of the Lie algebra on } \mathfrak{s u}(2)\end{array}$ \\
\hline$c$ & Constant angular velocity $(\mathrm{rad} / \mathrm{s})$ \\
\hline$C_{d}$ & Drag coefficient of spacecraft \\
\hline$F$ & $\begin{array}{l}\text { Isomorphism from Special Unitary } \\
\text { group } S U(2) \text { to the unit quater- } \\
\text { nions } \mathbb{H}\end{array}$ \\
\hline $\bar{h}_{w}$ & $\begin{array}{l}\text { Reaction wheel angular momentum } \\
\text { vector }\left(\mathrm{kgm}^{2} / \mathrm{s}\right)\end{array}$ \\
\hline$H$ & Rotational kinetic energy (Joules) \\
\hline$\hat{i}, \hat{j}, \hat{k}$ & Basis of the body reference frame \\
\hline$\hat{I}, \hat{J}, \hat{K}$ & $\begin{array}{l}\text { Basis of the Geocentric Equatorial } \\
\text { reference frame }\end{array}$ \\
\hline$I_{N}$ & $\begin{array}{l}\text { Accumulated torque of reaction } \\
\text { wheels }(N m s)\end{array}$ \\
\hline$J$ & Inertia matrix of spacecraft $\left(\mathrm{kgm}^{2}\right)$ \\
\hline$J_{s}$ & $\begin{array}{l}\text { Symmetric moment of inertia of } \\
\text { spacecraft }\left(\mathrm{kgm}^{2}\right)\end{array}$ \\
\hline$J_{w}$ & $\begin{array}{l}\text { Moment of inertia of reaction } \\
\text { wheels }\left(\mathrm{kgm}^{2}\right)\end{array}$ \\
\hline$K_{\omega}, K_{q}$ & Gain matrices \\
\hline$L$ & Lax Pair operator \\
\hline$M$ & $\begin{array}{l}\text { Magnitude of angular momentum } \\
\text { of spacecraft }\left(\mathrm{kgm}^{2} / \mathrm{s}\right)\end{array}$ \\
\hline$M_{r z}$ & $\begin{array}{l}\text { Residual dipole of spacecraft in } z \\
\text { body axis }\left(A m^{2}\right)\end{array}$ \\
\hline$\hat{n}_{c}$ & Unit vector of cone centreline \\
\hline$\hat{n}_{p}$ & $\begin{array}{l}\text { Unit vector of spacecraft body } \mathrm{x} \text { - } \\
\text { axis }\end{array}$ \\
\hline
\end{tabular}

Corresponding author. E-mail: craig.maclean@strath.ac.uk

\begin{tabular}{|c|c|}
\hline $\bar{N}_{e}$ & External torque vector $(\mathrm{Nm})$ \\
\hline $\bar{N}_{w}$ & Reaction wheel torque vector $(\mathrm{Nm})$ \\
\hline$p_{1}, p_{2}, p_{3}$ & $\begin{array}{l}\text { Increments to optimal angular ve- } \\
\text { locities }(\mathrm{rad} / \mathrm{s})\end{array}$ \\
\hline $\bar{q}$ & Quaternion vector \\
\hline $\bar{q}_{d}$ & Desired quaternion vector \\
\hline $\bar{q}_{e}$ & Quaternion error vector \\
\hline $\bar{r}_{C o P / C o M}$ & $\begin{array}{l}\text { Centre of mass to centre of pres- } \\
\text { sure vector }(m)\end{array}$ \\
\hline$\hat{R}, \hat{T}, \hat{N}$ & Basis of the RTN reference frame \\
\hline$R(t)$ & Rotation matrix on $S U(2)$ \\
\hline $\bar{u}$ & Control signal vector \\
\hline$T$ & Manoeuvre time (seconds) \\
\hline$\delta_{c}$ & Half angle of obstacle cone $(\mathrm{rad})$ \\
\hline$\delta_{p}$ & $\begin{array}{l}\text { Angle between spacecraft body } \mathrm{x} \text { - } \\
\text { axis and cone centreline unit vec- } \\
\text { tors }(\mathrm{rad})\end{array}$ \\
\hline$\rho$ & Reflectivity of spacecraft \\
\hline$\tau$ & Stabilisation time (seconds) \\
\hline$\varphi_{1}, \varphi_{2}, \varphi_{3}$ & Euler angles $(\mathrm{rad})$ \\
\hline $\bar{\omega}$ & $\begin{array}{l}\text { Inertially referenced body rate vec- } \\
\text { tor }(\mathrm{rad} / \mathrm{s})\end{array}$ \\
\hline $\bar{\omega}_{e}$ & $\begin{array}{l}\text { Angular velocity error vector } \\
(\mathrm{rad} / \mathrm{s})\end{array}$ \\
\hline $\bar{\omega}_{w}$ & $\begin{array}{l}\text { Reaction wheel angular velocity } \\
\text { vector }(\mathrm{rad} / \mathrm{s})\end{array}$ \\
\hline$\Omega$ & $\begin{array}{l}\text { Skew symmetric angular velocity } \\
\text { matrix }\end{array}$ \\
\hline
\end{tabular}

\section{INTRODUCTION}

Small spacecraft, such as UKube-1, are limited both in the size of their actuators and in their on-board computational capacity. As a result a low torque, computationally efficient means of repointing these nano-spacecraft is required.

In this paper an analytical motion planning algorithm for axisymmetric and asymmetric rigid spacecraft, based around the natural motion of a free rigid body, is derived in the framework of geometric mechanics and applied to the attitude control of nano-spacecraft in order to assess its effectiveness and the feasibility of future on-board implementation.

The attitude control of a rigid body has been widely studied. Initial approaches focussed on a series of discrete single axis slews. However these methods were costly in terms of time and fuel and so new methods were sought. Open loop controls [1], [2] were proposed as simple, low-cost solutions for spacecraft, but these methods are highly sensitive to uncertainties and disturbances. Despite this, open loop controls may be suitable for spacecraft attitude control in low disturbance environments 
with coarse pointing requirements [3]. Meanwhile Sidi [4] proposed augmenting single-axis open loop "bang-off-bang" controls with linear feedback near the origin, thus maintaining relatively low control effort while improving stability. Shortest angular path eigenaxis slews were proposed as simple, near time-optimal solutions [5], [6], [7], but they are in general not fuel-optimal. Other time-efficient retargeting methods have been proposed by Wie [8] and Verbin [9], which additionally consider actuator and pointing constraints. Nonlinear optimal control methods that use the calculus of variations and dynamic programming have also been applied to the spacecraft attitude control problem. However while these methods, such as those that require the numerical solution of the HamiltonJacobi-Bellman (HJB) equation, give globally optimal and theoretically superior solutions they are difficult to implement onboard a spacecraft [10] as they are computationally intensive. As a result, simple proportional-derivative (PD) controllers such as the quaternion feedback controllers [11], [12] continue to dominate the practical control of spacecraft due to the ease of gain tuning and low implementation risk. Recent inverse optimal control methods negate the need to solve the HJB equation by finding a control Lyapunov function which is itself a solution of the HJB, and designing the control around this [13]. This approach has been combined with the minimisation of control norms to form low energy attitude manoeuvres [14], [15]. Other guidance methods include the tracking of references generated by computationally efficient artificial potential functions (APFs) to reorientate spacecraft [16]. Mengali [17] extended this work by utilising artificial potential functions in the manoeuvring of spacecraft with sensitive optical equipment such as star trackers, which must reach the target attitude while avoiding luminous objects such as the Sun and Earth's Moon.

This work is motivated by the need to find a compromise between the computational simplicity of proportional-derivative control and the optimality of non-linear controllers, in an extension of the work presented in [18], [19]. In free space and choosing appropriate initial conditions, the spacecraft will naturally drift towards a certain orientation in a set period of time. Therefore these "natural motions" are an intuitive basis around which to design spacecraft attitude manoeuvres. In this paper the dynamics of a rigid body are exploited to derive analytical expressions for the time evolution of the attitude of the spacecraft in quaternion form. Despite suffering from the problem of ambiguity, quaternions are singularity free and computationally efficient, and so are the most widely used form of attitude representation on-board spacecraft [20]. Boundary conditions on the attitude of the spacecraft are matched through parametric optimisation of the initial spacecraft angular velocities, and the resulting references are tracked using a simple proportional-derivative controller. It is shown via comparison with a quaternion feedback control that the references require low accumulated torque to track. In addition, the set of curves which match the boundary conditions on the final pointing direction can be probed to create a simple obstacle avoidance algorithm.

The main contributions of the paper are:
1) While the classical global solution is on the attitude dynamics, in this paper the kinematics for both axisymmetric and asymmetric spacecraft are derived analytically for global motion planning implementation in convenient quaternion form. The classical solutions, to the authors' knowledge, can only be found expressed locally in terms of Euler angles [21], [22], [23].

2) The analytical equations are implemented in a simple motion planning algorithm to produce easily trackable reference motions for a spacecraft, and it is shown that the motion planning algorithm is additionally capable of providing constrained slews.

3) The strengths and weaknesses of the proposed method are evaluated through extensive numerical simulations, and it is shown that the natural motions provide substantial savings in accumulated torque over a conventional quaternion feedback benchmark.

The paper is structured as follows. In Section II, the spacecraft model is introduced, including the alternative kinematics on the Lie group $S U(2)$ which enable a more elegant solution to the free body motion to be derived. In Section III, the general motion planning framework is first described, before the specific cases of the axisymmetric and asymmetric spacecraft are derived. The practical implementation of the motion planning method is discussed in Section IV, including the extension to obstacle avoidance. Simulation results are presented in Section $\mathrm{V}$ where the natural motions are compared to conventional quaternion feedback control, and the suitability of the method for on-board implementation is discussed in Section VI. Finally, we conclude with Section VII and a discussion of areas of future research.

\section{MODELS}

While in reality spacecraft have flexible modes, in this paper the assumption is made that the spacecraft can be treated as a rigid body. This assumption is feasible for small Cubesat spacecraft as they contain few moving parts and generally do not carry liquid propellant. The general equations describing the attitude control problem are then that of a rigid body with external torques describing the effect of the disturbances.

\section{A. Reference Frame Definitions}

The spacecraft under consideration is in orbit around the Earth. The centre of the Earth is chosen as the origin of a Geocentric Equatorial co-ordinate system with basis vectors $\hat{I}, \hat{J}, \hat{K}$. As in [24], the $\mathrm{X}$-axis lies in the equatorial plane towards the vernal equinox direction, the $\mathrm{Z}$-axis points in the direction of the North pole and the Y-axis lies in the equatorial plane and completes the orthonormal reference frame. The coordinate system is shown in Figure 1. 


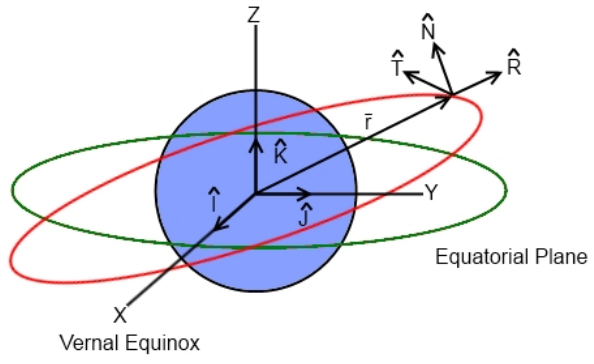

Figure 1: Geocentric Equatorial and RTN co-ordinate systems.

Also shown in Figure 1 is the Radial-Transverse-Normal (RTN) reference frame used to describe the orbit of the spacecraft. In this reference frame $\hat{R}$ is parallel with the radial vector, $\hat{N}$ is parallel with the orbit normal and $\hat{T}$ completes the orthonormal frame.

A body fixed reference frame (BRF) with basis $\hat{i}, \hat{j}, \hat{k}$ is rigidly attached to the centre of mass of the spacecraft, as shown in Figure 2.

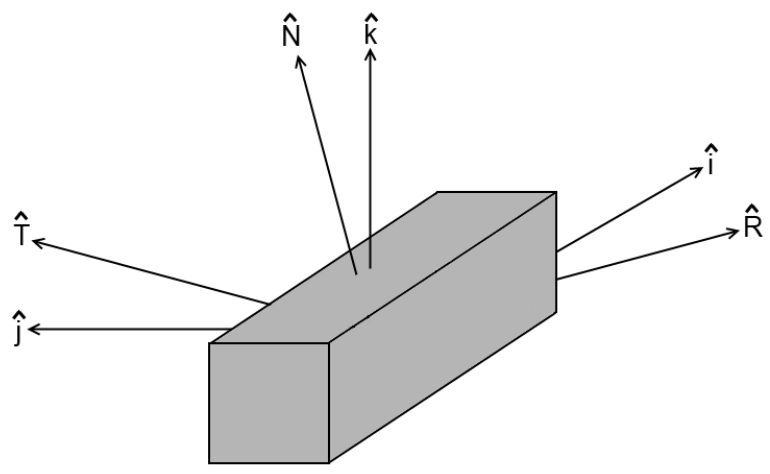

Figure 2: Body and RTN co-ordinate systems.

\section{B. Kinematic Model}

The attitude kinematics of the spacecraft can be parameterised using quaternions:

$$
\frac{d \bar{q}}{d t}=\frac{1}{2} \Omega \bar{q}
$$

where $\bar{q}=\left[\begin{array}{llll}q_{0} & q_{1} & q_{2} & q_{3}\end{array}\right]^{T}$ denotes the quaternions which represent the attitude of the spacecraft in the body frame with respect to the inertial frame, and $\frac{d \bar{q}}{d t}$ their rate of change. The skew symmetric matrix $\Omega$ is given by:

$$
\Omega=\left(\begin{array}{cccc}
0 & -\omega_{1} & -\omega_{2} & -\omega_{3} \\
\omega_{1} & 0 & \omega_{3} & -\omega_{2} \\
\omega_{2} & -\omega_{3} & 0 & \omega_{1} \\
\omega_{3} & \omega_{2} & -\omega_{1} & 0
\end{array}\right)
$$

where $\bar{\omega}=\left[\begin{array}{lll}\omega_{1} & \omega_{2} & \omega_{3}\end{array}\right]^{T}$ are the angular velocities of the spacecraft body frame with respect to the inertial frame. The quaternions must satisfy the constraint $q_{0}^{2}+q_{1}^{2}+q_{2}^{2}+q_{3}^{2}=1$. The quaternion differential equations are used as they do not suffer from problems with singularities or imaginary numbers. This representation is equivalent to the kinematic matrix representation on the Special Unitary group $S U(2)$ :

$$
\frac{d R(t)}{d t}=R(t)\left(\omega_{1} A_{1}+\omega_{2} A_{2}+\omega_{3} A_{3}\right)
$$

where $R(t) \in S U(2)$ represents the orientation of the spacecraft and $A_{1}, A_{2}, A_{3}$ form a basis for the Lie algebra $\mathfrak{s u}(2)$ of the Lie group $S U(2)$ :

$$
\begin{aligned}
& A_{1}=\frac{1}{2}\left(\begin{array}{cc}
i & 0 \\
0 & -i
\end{array}\right) \\
& A_{2}=\frac{1}{2}\left(\begin{array}{cc}
0 & 1 \\
-1 & 0
\end{array}\right) \\
& A_{3}=\frac{1}{2}\left(\begin{array}{cc}
0 & i \\
i & 0
\end{array}\right)
\end{aligned}
$$

where $i$ is an imaginary number. The Lie algebra's commutator, called the Lie bracket, is defined by $[X, Y]=Y X-X Y$ with $X, Y \in \mathfrak{s u}(2)$ such that $\left[A_{1}, A_{2}\right]=A_{3},\left[A_{2}, A_{3}\right]=A_{1}$ and $\left[A_{1}, A_{3}\right]=-A_{2}$. The rotation matrix $R(t) \in S U(2)$ is of the form:

$$
R(t)=\left(\begin{array}{cc}
z_{1} & z_{2} \\
-\bar{z}_{2} & \bar{z}_{1}
\end{array}\right)
$$

with $z_{1}, z_{2} \in \mathbb{C}$ and $\bar{z}_{1}, \bar{z}_{2}$ their complex conjugates such that $\left|z_{1}\right|^{2}+\left|z_{2}\right|^{2}=1$. Physically the basis $A_{1}, A_{2}, A_{3}$ describe the infinitesimal motion of the spacecraft in the roll, pitch and yaw directions respectively.

Klein [25] discovered that for the symmetric Lagrange and toy top (a symmetric rigid body in a constant gravitational field) simpler solutions can be obtained when $S U(2)$ rather than $S O(3)$, the set of $3 \times 3$ rotation matrices, is used as the configuration space. Thus in this paper $S U(2)$ is used as the configuration space as it enables the equations of motion to be expressed and solved in convenient Lax pair form. In addition $S U(2)$ is isomorphic to the unit quaternions [26] which enables the solution to be expressed in quaternion form.

The two sets of kinematic equations (1) and (3) are equivalent with the isomorphism, $F: S U(2) \leftrightarrow \mathbb{H}$, from the Special Unitary group to the unit quaternions:

$$
F:\left(\begin{array}{cc}
z_{1} & z_{2} \\
-\bar{z}_{2} & \bar{z}_{1}
\end{array}\right) \leftrightarrow \mathbf{z}_{1}+\mathbf{z}_{2} \cdot \mathbf{j}=q_{0} \mathbf{e}+q_{1} \mathbf{i}+q_{2} \mathbf{j}+q_{3} \mathbf{k}
$$

defining the coordinate change. The complex numbers $z_{1}=q_{0}+i q_{1}, z_{2}=q_{2}+i q_{3}$ are regarded in their quaternion form $\mathbf{z}_{1}=q_{0} \mathbf{e}+q_{1} \mathbf{i}, \mathbf{z}_{2}=q_{2} \mathbf{e}+q_{3} \mathbf{i}$ subject to the usual quaternionic multiplication rules. For more details of this isomorphism see [27] pp. 169-171.

\section{Dynamic Model}

Euler's rotational equations of motion for a rigid spacecraft are defined as:

$$
J \cdot \dot{\bar{\omega}}+\bar{\omega} \times J \cdot \bar{\omega}=\bar{N}_{e}+\bar{N}_{w}
$$

where $J$ denotes the moment of inertia matrix of the spacecraft, $\bar{\omega}$ and $\dot{\bar{\omega}}$ the angular velocity and angular acceleration vectors 
Table I: Physical properties of spacecraft

\begin{tabular}{l|l}
\hline \hline Principal inertias of axisymmetric spacecraft A & $J_{1}=0.0109 \mathrm{kgm}^{2}$ \\
& $J_{2}=J_{3}=J_{s}=0.05 \mathrm{kgm}^{2}$ \\
Principal inertias of asymmetric spacecraft B & $J_{1}=0.0109 \mathrm{kgm}^{2}$ \\
& $J_{2}=0.0504 \mathrm{kgm}^{2}$ \\
& $J_{3}=0.0506 \mathrm{kgm}^{2}$ \\
Drag coefficient & $C_{d}=3$ \\
Reflectivity & $\rho=0.6$ \\
Residual dipole in z-body axis & $M_{r z}=10 \times 10^{-3} \mathrm{Am}^{2}$ \\
CoP/CoM offset & $\left\|\bar{r}_{C o P} / \mathrm{CoM}\right\|=0.02 \mathrm{~m}$ \\
\hline \hline
\end{tabular}

Table II: Reaction wheel data

\begin{tabular}{l|l}
\hline \hline Wheel inertias & $J_{w}=1.499 \times 10^{-5} \mathrm{kgm}^{2}$ \\
Maximum wheel torque & $1 \times 10^{-3} \mathrm{Nm}$ \\
Rate limit & $1 \times 10^{-2} \mathrm{Nm} / \mathrm{s}$ \\
Nominal wheel momentum & $7 \times 10^{-3} \mathrm{Nms} @ 4460 \mathrm{rpm}$ \\
\hline \hline
\end{tabular}

of the spacecraft body frame with respect to the inertial frame, $\bar{N}_{e}=\left[\begin{array}{lll}N_{1 e} & N_{2 e} & N_{3 e}\end{array}\right]^{T}$ the external torques and $\bar{N}_{w}=$ $\left[\begin{array}{lll}N_{1 w} & N_{2 w} & N_{3 w}\end{array}\right]^{T}$ the reaction wheel torques. Assuming that the body frame originates from the spacecraft centre of mass and is coincident with the principal axes of the spacecraft, Euler's equations reduce to:

$$
\begin{aligned}
& \dot{\omega}_{1}=\frac{N_{1 e}+N_{1 w}+\left(J_{2}-J_{3}\right) \omega_{2} \omega_{3}}{J_{1}} \\
& \dot{\omega}_{2}=\frac{N_{2 e}+N_{2 w}+\left(J_{3}-J_{1}\right) \omega_{3} \omega_{1}}{J_{2}} \\
& \dot{\omega}_{3}=\frac{N_{3 e}+N_{3 w}+\left(J_{1}-J_{2}\right) \omega_{1} \omega_{2}}{J_{3}}
\end{aligned}
$$

where $J_{1}, J_{2}$ and $J_{3}$ are the principal moments of inertia of the spacecraft.

\section{Spacecraft Model}

We primarily focus on two spacecraft based on the UKube1 ; one of which is axisymmetric and the other slightly asymmetric. The properties of these spacecraft are listed in Table I.

We assume that the spacecraft is equipped with simple reaction wheels. The wheel data is based on the Sinclair Interplanetary picosatellite reaction wheels ${ }^{1}$, and the wheel properties are shown in Table II. The wheels apply a control torque:

$$
N_{w}=-\dot{\bar{h}}_{w}-\bar{\omega} \times \bar{h}_{w}
$$

This simplified model can be easily integrated to yield the wheel angular momenta $\bar{h}_{w}=\left[\begin{array}{lll}h_{1 w} & h_{2 w} & h_{3 w}\end{array}\right]^{T}$ and wheel velocities $\bar{\omega}_{w}=\left[\begin{array}{lll}\omega_{1 w} & \omega_{2 w} & \omega_{3 w}\end{array}\right]^{T}$.

\section{E. Environmental Model}

The spacecraft is subjected to disturbance torques due to gravity gradient [20], SRP and air drag [28], and the residual dipole of the spacecraft ${ }^{2}$. The magnetic field is modelled using a simple dipole model [29], rotated to mirror the offset between the geographic and geomagnetic poles. The spacecraft is considered to be on a $600 \mathrm{~km}$ altitude circular orbit beginning at the vernal equinox position, unless stated otherwise.

The environmental model is required to assess the practical performance of the natural motions, derived subsequently for the free rigid body in Section III, when applied to a spacecraft in a non-ideal environment.

\section{ANALYTIC DERIVATION OF THE REFERENCE MOTIONS}

In this section analytical equations for the torque-free attitude motion of axisymmetric and asymmetric spacecraft are derived using Lax Pair integration in global quaternion form. The general motion planning framework will first be described, before the specific cases of the axisymmetric and asymmetric spacecraft are considered. These analytical equations will form the basis of the natural motion planning algorithm for spacecraft attitude manoeuvres.

\section{A. General Framework}

Firstly, we note that in the absence of external torques the following quantities are constant:

$$
\begin{aligned}
& H=\frac{1}{2}\left(J_{1} \omega_{1}^{2}+J_{2} \omega_{2}^{2}+J_{3} \omega_{3}^{2}\right) \\
& M^{2}=\left(J_{1} \omega_{1}\right)^{2}+\left(J_{2} \omega_{2}\right)^{2}+\left(J_{3} \omega_{3}\right)^{2}
\end{aligned}
$$

These correspond to the Hamiltonian, in this case the total rotational kinetic energy of the spacecraft, and the Casimir function, in this case the magnitude of the angular momentum of the spacecraft.

In order to derive the global solution in terms of quaternions, we utilise the local solution in terms of euler angles.

Lemma 1. The euler angles representing the local orientation of the spacecraft can be written as functions of the angular velocities [21], [22], [23]:

$$
\begin{aligned}
& \varphi_{1}=\int \frac{M\left(2 H-J_{1} \omega_{1}^{2}\right)}{M^{2}-\left(J_{1} \omega_{1}\right)^{2}} d t \\
& \varphi_{2}=\arccos \left(\frac{J_{1} \omega_{1}}{M}\right) \\
& \varphi_{3}=\arctan \left(\frac{J_{2} \omega_{2}}{J_{3} \omega_{3}}\right)
\end{aligned}
$$

where $0 \leq \varphi_{2} \leq \pi$ and $-\pi / 2<\varphi_{3}<\pi / 2$.

Proof. The Euler equations (7) can be written in Lax pair form on $S U(2)$ as [30], [31]:

$$
\frac{d L(t)}{d t}=[L(t), \omega]
$$

where:

$$
\begin{gathered}
L(t)=J_{1} \omega_{1} A_{1}+J_{2} \omega_{2} A_{2}+J_{3} \omega_{3} A_{3} \\
\omega=\omega_{1} A_{1}+\omega_{2} A_{2}+\omega_{3} A_{3}
\end{gathered}
$$

It is well known that the Lax Pair equation and the kinematic equations are connected through the relation:

$$
L(t)=R(t)^{-1} L(0) R(t)
$$

\footnotetext{
${ }^{1}$ http://www.sinclairinterplanetary.com/reactionwheels

${ }^{2}$ http://www.dept.aoe.vt.edu/cdhall/courses/aoe4065/NASADesignSPs/sp8018.pdf
} 
and it is this relation that we use to solve for the corresponding rotations. Here $L(0)$ is the matrix $L(t)$ at $t=0$ and is therefore a matrix with constant entries. Writing the dynamics and kinematics in the language of classical mechanics and making use of Lax pair integration provides a natural and elegant means of deriving analytically the expressions for the time evolution of the quaternions. Equivalently we can write:

$$
R(t) L(t) R(t)^{-1}=L(0)
$$

where $R(t) L(t) R(t)^{-1}$ describes the conjugacy class of $L(t)$. Thus for simplicity and to obtain more explicit solutions it suffices to integrate the particular solution:

$$
R(t) L(t) R(t)^{-1}=M A_{1}
$$

where $M$ is the conserved Casimir function from (10). This enables us to write:

$$
L(t)=M R(t)^{-1} A_{1} R(t)
$$

As $\exp \left(-\varphi_{1} A_{1}\right) A_{1} \exp \left(\varphi_{1} A_{1}\right)=A_{1}$, we say that $\exp \left(\varphi_{1} A_{1}\right)$ is the stabilizer of $A_{1}$ [27]. It is therefore convenient to introduce the coordinate form:

$$
R(t)=\exp \left(\varphi_{1} A_{1}\right) \exp \left(\varphi_{2} A_{2}\right) \exp \left(\varphi_{3} A_{1}\right)
$$

Substituting (18) into (17) yields:

$$
L(t)=\frac{i M}{2}\left(\begin{array}{cc}
\cos \varphi_{2} & e^{-i \varphi_{3}} \sin \varphi_{2} \\
e^{i \varphi_{3}} \sin \varphi_{2} & -\cos \varphi_{2}
\end{array}\right)
$$

Equating (19) with $L(t)$ in (13) results in the following system of equations:

$$
\begin{aligned}
& J_{1} \omega_{1}=M \cos \varphi_{2} \\
& J_{2} \omega_{2}+i J_{3} \omega_{3}=M e^{-i \varphi_{3}} \sin \varphi_{2} \\
& -J_{2} \omega_{2}+i J_{3} \omega_{3}=M e^{i \varphi_{3}} \sin \varphi_{2}
\end{aligned}
$$

which can be immediately solved to provide the expression for $\varphi_{2}$ in (11). Now, adding the bottom two equations in (20) yields:

$$
2 i J_{3} \omega_{3}=M \sin \varphi_{2}\left(e^{-i \varphi_{3}}+e^{i \varphi_{3}}\right)
$$

While subtracting the bottom two equations in (20) gives:

$$
2 J_{2} \omega_{2}=-M \sin \varphi_{2}\left(-e^{-i \varphi_{3}}+e^{i \varphi_{3}}\right)
$$

Equations (21) and (22) can be simplified by making use of the identities:

$$
\begin{aligned}
& \cos (\theta)=\frac{e^{i \theta}+e^{-i \theta}}{2} \\
& \sin (\theta)=\frac{e^{i \theta}-e^{-i \theta}}{2 i}
\end{aligned}
$$

which results in the following:

$$
\begin{gathered}
i J_{3} \omega_{3}=M \sin \left(\varphi_{2}\right) \cos \left(\varphi_{3}\right) \\
\frac{J_{2} \omega_{2}}{i}=-M \sin \left(\varphi_{2}\right) \sin \left(\varphi_{3}\right)
\end{gathered}
$$

Dividing these two equations we obtain:

$$
\tan \left(\varphi_{3}\right)=\frac{J_{2} \omega_{2}}{J_{3} \omega_{3}}
$$

Solving, we arrive at the expression for $\varphi_{3}$ in (11). To obtain an expression for $\varphi_{1}$, we first rearrange (3) to yield:

$$
R(t)^{-1} \frac{d R(t)}{d t}=\omega_{1} A_{1}+\omega_{2} A_{2}+\omega_{3} A_{3}
$$

Then, substituting the expressions for $\varphi_{2}$ and $\varphi_{3}$ into (18), and subsequently into (26), with some computation we find that:

$$
\dot{\varphi}_{1}=\frac{M \omega_{1}\left(M^{2}-\left(J_{1} \omega_{1}\right)^{2}\right)-M J_{2} J_{3}\left(\omega_{3} \dot{\omega}_{2}-\omega_{2} \dot{\omega}_{3}\right)}{J_{1} \omega_{1}\left(M^{2}-\left(J_{1} \omega_{1}\right)^{2}\right)}
$$

Using the expressions for $\dot{\omega}_{2}$ and $\dot{\omega}_{3}$ in (7) for the free body (i.e. $\bar{N}_{e}=\bar{N}_{w}=0$ ), the constants (10) and simplifying we arrive at the expression for $\varphi_{1}$ in (11).

Substituting (11) into (18) and pulling the solution back to the identity via:

$$
R(t)=R_{\text {int }} R(0)^{-1} R(t)
$$

provides the solution on the Special Unitary group $S U(2)$. In this expression $R_{\text {int }}$ is the initial orientation and $R(0)^{-1}$ is the inverse of $R(t)$ at $t=0$. Finally, using the isomorphism (6) and comparing the real and imaginary parts yields the globally defined analytical quaternion equations for the free motion of a rigid body.

We now utilise the equations above to derive the globally defined analytic equations for the free motion of the spacecraft in the axisymmetric and asymmetric cases.

\section{B. Axisymmetric Spacecraft}

In this subsection we state the equations for the free motion of an axisymmetric spacecraft, derived using the procedure in Section III-A.

Lemma 2. The time evolution of the quaternions $(\bar{q}=$ $\left[\begin{array}{llll}q_{0} & q_{1} & q_{2} & q_{3}\end{array}\right]^{T}$ ) related to the free motion of an axisymmetric body can be written in the form:

$$
\begin{aligned}
& q_{0}=\cos (x(t)) \cos \left(\frac{\varphi_{1}}{2}\right)-y(t) \sin (x(t)) \sin \left(\frac{\varphi_{1}}{2}\right) \\
& q_{1}=\sin (x(t)) \cos \left(\frac{\varphi_{1}}{2}\right)+y(t) \cos (x(t)) \sin \left(\frac{\varphi_{1}}{2}\right) \\
& q_{2}=\operatorname{sgn}\left(\omega_{3}(0)\right) \sqrt{1-y(t)^{2}} \sin \left(\frac{\varphi_{1}}{2}\right) \sin (z(t)) \\
& q_{3}=\operatorname{sgn}\left(\omega_{3}(0)\right) \sqrt{1-y(t)^{2}} \sin \left(\frac{\varphi_{1}}{2}\right) \cos (z(t))
\end{aligned}
$$

where:

$$
\begin{aligned}
& x(t)=\frac{c\left(J_{s}-J_{1}\right)}{2 J_{s}} t \\
& y(t)=\frac{J_{1} c}{M} \\
& z(t)=x(t)+\tan ^{-1}\left(\frac{\omega_{2}(0)}{\omega_{3}(0)}\right) \\
& \varphi_{1}=\left(M\left(2 H-J_{1} c^{2}\right) /\left(M^{2}-\left(J_{1} c\right)^{2}\right) t\right.
\end{aligned}
$$

Proof. The analytical expressions for the angular velocities of an axisymmetric spacecraft, where $J_{2}=J_{3}=J_{s}$, are well known and are given by [20]:

$$
\begin{aligned}
& \omega_{1}=c \\
& \omega_{2}=\operatorname{sgn}\left[\omega_{3}(0)\right] \sigma \sin \left(\lambda t+\tan ^{-1}\left(\frac{\omega_{2}(0)}{\omega_{3}(0)}\right)\right) \\
& \omega_{3}=\operatorname{sgn}\left[\omega_{3}(0)\right] \sigma \cos \left(\lambda t+\tan ^{-1}\left(\frac{\omega_{2}(0)}{\omega_{3}(0)}\right)\right)
\end{aligned}
$$

where:

$$
\begin{aligned}
& \lambda=c \frac{J_{1}-J_{s}}{J_{s}} \\
& \sigma^{2}=\frac{2 H-J_{1} c^{2}}{J_{s}}
\end{aligned}
$$


Substituting these expressions into (11) and then into (18), and pulling the solution back to the identity via (28) yields the solution on $S U(2)$. Using the isomorphism (6) gives the expressions for the evolution of the quaternions in the axisymmetric case $\square$ See [18]

Remark 1. The sign function $\operatorname{sgn}\left(\omega_{3}(0)\right)$ in (29) and (31) is added to ensure that the initial first derivatives of these equations match the initial first derivatives of their numerical counterparts. That is, to ensure that the first derivatives of (29) and (31) at time $t=0$ match the first derivatives of (1) and (7), with zero external torques, at $t=0$.

\section{Asymmetric Spacecraft}

In this subsection we state the equations for the free motion of an asymmetric spacecraft, derived using the procedure in Section III-A.

Lemma 3. The time evolution of the quaternions $(\bar{q}=$ $\left.\left[\begin{array}{llll}q_{0} & q_{1} & q_{2} & q_{3}\end{array}\right]^{T}\right)$ related to the free motion of an asymmetric body can be written in the form:

$$
\begin{aligned}
& q_{0}=\mathscr{F}_{1}\left(\cos \left(\frac{\varphi_{1}}{2}\right) \mathscr{F}_{3}-\sin \left(\frac{\varphi_{1}}{2}\right) \mathscr{F}_{4}\right) \\
& q_{1}=\mathscr{F}_{1}\left(\sin \left(\frac{\varphi_{1}}{2}\right) \mathscr{F}_{3}+\cos \left(\frac{\varphi_{1}}{2}\right) \mathscr{F}_{4}\right) \\
& q_{2}=\mathscr{F}_{2}\left(\cos \left(\frac{\varphi_{1}}{2}\right) \mathscr{F}_{3}+\sin \left(\frac{\varphi_{1}}{2}\right) \mathscr{F}_{4}\right) \\
& q_{3}=\mathscr{F}_{2}\left(\sin \left(\frac{\varphi_{1}}{2}\right) \mathscr{F}_{3}-\cos \left(\frac{\varphi_{1}}{2}\right) \mathscr{F}_{4}\right)
\end{aligned}
$$

where

$$
\begin{aligned}
& \varphi_{1}=\frac{M}{J_{1}} t+\kappa \Pi(n ; \vartheta \mid m)+D, \\
& \mathscr{F}_{1}=\mathscr{S}_{1} \sqrt{\frac{1+x(t)}{2}}, \\
& \mathscr{F}_{2}=\mathscr{S}_{2} \sqrt{\frac{1-x(t)}{2}}, \\
& \mathscr{F}_{3}=\mathscr{S}_{3} \frac{1}{\sqrt{1+y^{\star}(t)^{2}}}, \\
& \mathscr{F}_{4}=\mathscr{S}_{4} \frac{y^{\star}(t)^{\star}}{\sqrt{1+y^{\star}(t)^{2}}}, \\
& \mathscr{J}_{i}= \pm 1, \text { for } i=1,2,3,4, \\
& y^{\star}(t)=\frac{y(t)}{1+\sqrt{1+y(t)^{2}}}, \\
& x(t)=\frac{J_{1} \omega_{1}}{M} \\
& y(t)=\frac{J_{2} \omega_{2}}{J_{3} \omega_{3}} .
\end{aligned}
$$

where $\Pi(n ; \vartheta \mid m)$ is the incomplete elliptic integral of the third kind with

$$
\begin{aligned}
& n=s_{1} / M^{2} \\
& f(t)= \pm \sqrt{s_{2} \alpha} t+C_{11} \\
& m=s_{1} / s_{2} \\
& \vartheta=a m(f(t), m) \\
& \kappa= \pm\left(2 H J_{1}-M^{2}\right) /\left(M J_{1} \sqrt{s_{2} \alpha}\right)
\end{aligned}
$$

when $\left|s_{1} / s_{2}\right| \leq 1$. When $\left|s_{1} / s_{2}\right|>1$ we have that:

$$
\begin{aligned}
& n=s_{2} / M^{2} \\
& f(t)= \pm \sqrt{s_{1} \alpha} t+\sqrt{\frac{s_{1}}{s_{2}}} C_{11} \\
& m=s_{2} / s_{1} \\
& \vartheta=a m(f(t), m) \\
& \kappa= \pm\left(2 H J_{1}-M^{2}\right) /\left(M J_{1} \sqrt{s_{1} \alpha}\right)
\end{aligned}
$$

where $\operatorname{am}(\cdot, m)$ is the Jacobi amplitude, $D$ is a constant of integration and $\omega_{1}, \omega_{2}, \omega_{3}$ are the free rigid body angular velocities.

Proof. The free rigid body angular velocities $\omega_{i}$ can be expressed in the analytic form [26], [32]:

$$
\omega_{i}=\frac{\sqrt{s_{i}}}{J_{i}} \operatorname{sn}\left( \pm \sqrt{\alpha s_{j}} t+C_{i}, \frac{s_{i}}{s_{j}}\right)
$$

when

$$
\left|\frac{s_{i}}{s_{j}}\right| \leq 1
$$

or

$$
\omega_{i}=\frac{\sqrt{s_{j}}}{J_{i}} s n\left( \pm \sqrt{\alpha s_{i}} t+\sqrt{\frac{s_{i}}{s_{j}}} C_{i}, \frac{s_{j}}{s_{i}}\right)
$$

otherwise ${ }^{3}$.

The function $\operatorname{sn}(\cdot, \cdot)$ is a Jacobi elliptic function and the constants $C_{i}$ are defined by

$$
C_{i}=s n^{-1}\left(\frac{J_{i} \omega_{i}(0)}{\sqrt{s_{i}}}, \frac{s_{i}}{s_{j}}\right)
$$

with

$$
s_{i}=\frac{-\beta+\sqrt{\beta^{2}-4 \alpha \chi}}{2 \alpha} \quad s_{j}=\frac{-\beta-\sqrt{\beta^{2}-4 \alpha \chi}}{2 \alpha}
$$

and

$$
\begin{aligned}
& \alpha=-\frac{\left(J_{i}-J_{j}\right)\left(J_{i}-J_{k}\right)}{J_{j} J_{k}} \\
& \beta=\frac{4 J_{j} J_{k} H-2 J_{i}\left(J_{j}+J_{k}\right) H+2 J_{i} M^{2}-\left(J_{j}+J_{k}\right) M^{2}}{J_{i} J_{j} J_{k}} \\
& \chi=-\frac{\left(2 J_{j} H-M^{2}\right)\left(2 J_{k} H-M^{2}\right)}{J_{i}^{2} J_{j} J_{k}}
\end{aligned}
$$

where the indexes do not represent a sum; $i, j$ and $k$ follow a "circular notation", which means they appear in a consecutive recursion (e.g. $i=1, j=2, k=3$ or $i=2, j=3, k=1$ etc. ...) See [32]. Substituting these expressions into (11) and then (18), and pulling the solution back to the identity via (28) yields the solution on $S U(2)$. Using the isomorphism (6) gives the expressions for the evolution of the quaternions in the axisymmetric case $\square$ See [19].

Remark 2. The sign \pm in (37) is dependent on the initial conditions. For implementation the sign has to be chosen so that the sign of the first derivative of (37) at the initial time $t=0$ :

$$
\dot{\omega}_{i}(0)= \pm \sqrt{\alpha s_{j}} \frac{\sqrt{s_{i}}}{J_{i}} c n\left(C_{i}, \frac{s_{i}}{s_{j}}\right) d n\left(C_{i}, \frac{s_{i}}{s_{j}}\right)
$$

${ }^{3}$ Using the relation:

$$
\sqrt{\mu} \operatorname{sn}(\zeta, \mu)=\operatorname{sn}\left(\sqrt{\mu} \zeta, \mu^{-1}\right)
$$


is matched to the sign of the Euler equations (7), with zero external torques, at $t=0$.

Remark 3. The $\mathscr{S}_{i}$ functions are sign functions. To implement the equations it is enough to consider only $\mathscr{S}_{1}$ or $\mathscr{S}_{2}$ and $\mathscr{S}_{3}$ or $\mathscr{S}_{4}$ respectively. The sign functions can be set by comparison with the known initial first derivative of the quaternions (1).

\section{Practical Implementation of Motion Planning METHOD}

With the analytical equations for the free motion of an axisymmetric and an asymmetric rigid body derived in quaternion form, we now construct a method to utilise these solutions in a motion planning algorithm. This requires the specification of an appropriate cost function and the implementation of some means of optimising the free parameters of the analytical solutions to match the set boundary conditions. A method of adapting the motion planning method to yield simple constrained manoeuvres is also presented.

\section{A. Parametric Optimisation}

In order to utilise the analytical expressions for the angular velocities and the globally defined quaternions for motion planning, the equations were entered into the program Mathematica. A cost function of the form:

$$
\min _{\bar{\omega}(0)}\{\|(\bar{q}-\bar{q}(T))\|\}
$$

was constructed, where $\bar{\omega}(0)$ are the initial angular velocities, $\bar{q}$ is the current quaternion value, $\bar{q}(T)$ is the target quaternion at time $t=T$ and $T$ is the manoeuvre time. This cost function minimises the norm of the error between the current quaternion and the target quaternion by changing the initial angular velocities. It follows that a parametric optimisation can be carried out to find the values of the initial angular velocities $\bar{\omega}(0)$ required to bring the spacecraft to the $\operatorname{target} \bar{q}(T)$ in a fixed time.

The inbuilt Mathematica direct search method "DifferentialEvolution", was used for the parametric optimisation as it proved the most effective at minimising the final pointing error in comparison to the other inbuilt solvers "RandomSolution", "SimulatedAnnealing" and "NelderMead". This method was chosen in order to assess the suitability of using the natural motions of the rigid body as the basis for designing attitude motions. A deeper investigation would be required to identify the most suitable method of determining the required initial angular velocities if the references were to be generated onboard a nano-spacecraft. However this is outwith the scope of this paper.

The optimal values of the initial angular velocities, $\bar{\omega}^{*}(0)$, resulting from the optimisation can then be input into the analytical equations for the angular velocities and quaternions to generate the reference tracks for a natural motion manoeuvre.

\section{B. Extension to constrained repointing}

Often a spacecraft is required to perform a constrained slew: that is, to repoint while avoiding or tracking certain objects. For example camera lenses can be damaged if they are pointed directly at a bright object such as the Sun or the Earth's Moon, while in contrast for power generation purposes it may be necessary to maintain a certain minimum angle between a solar array and the Sun throughout a manoeuvre. By probing the set of the natural motions which satisfy the boundary conditions on the final orientation of the spacecraft, $\bar{q}(T)$, the method proposed in Section IV-A can be easily extended to achieve such constrained slews.

Assuming that a camera is mounted along the spacecraft body $\mathrm{x}$-axis, a bright object such as the Sun creates a "cone" which the $\mathrm{x}$-axis unit vector must not intercept during a repointing manoeuvre. This constraint can be formalised as [17]:

$$
\delta_{p} \triangleq \arccos \left(\hat{n}_{p} \cdot \hat{n}_{c}\right)>\delta_{c}
$$

That is, the angle $\delta_{p}$ between the unit vectors of the spacecraft body $\mathrm{x}$-axis, $\hat{n}_{p}$, and the centreline of the cone, $\hat{n}_{c}$, must be greater than the half-angle of the cone $\delta_{c}$ throughout the manoeuvre.

Given the proposed method outlined above in Section IV-A, without a constraint on the pointing direction the parametric optimisation will return the optimal initial angular velocities $\omega_{1}{ }^{*}(0), \omega_{2}{ }^{*}(0), \omega_{3}{ }^{*}(0)$. In the case that this returns a motion that intersects a forbidden region $\left(\delta_{p}<\delta_{c}\right.$ for any $t \in[0, T]$ ) we introduce the parameters $p_{1}, p_{2}, p_{3}$ as increments to the angular velocities $\omega_{1}{ }^{*}(0), \omega_{2}{ }^{*}(0), \omega_{3}{ }^{*}(0)$, where $-\varepsilon<$ $p_{1}, p_{2}, p_{3}<\varepsilon$ and $\varepsilon$ is a parameter which can be tuned. We then optimise the objective function:

$$
\min _{p_{1}, p_{2}, p_{3}}\{\|(\bar{q}-\bar{q}(T))\|\}
$$

to find an alternative natural motion manoeuvre from $\bar{q}$ to $\bar{q}(T)$. The parameter $\varepsilon$ is then incremented iteratively to obtain a solution such that $\delta_{p}>\delta_{c} \forall t$. This leads to a new set of initial angular velocities $\omega_{1}{ }^{* *}(0)=\omega_{1}{ }^{*}(0)+p_{1}, \omega_{2}{ }^{* *}(0)=$ $\omega_{2}{ }^{*}(0)+p_{2}, \omega_{3}{ }^{* *}(0)=\omega_{3}^{*}(0)+p_{3}$ being obtained, which bring the spacecraft from $\bar{q}$ to $\bar{q}(T)$ with $\delta_{p}>\delta_{c} \forall t$. These angular velocities can then be input into the analytical expressions for the angular velocities and quaternions to generate reference tracks for the constrained natural motion manoeuvre.

\section{Natural Motion Planning Simulations}

The natural motion planning method described above was tested in simulation to assess the strengths and limitations of the method. As proportional-derivative (PD) controllers are the most commonly used means of carrying out repointing manoeuvres due to their simplicity and ease of gain tuning, a PD controller [11], [14] was chosen as a benchmark:

$$
\bar{u}=-K_{\omega} \bar{\omega}_{e}-K_{q} \bar{q}_{e}
$$

In this expression $\bar{u}$ is the desired control signal and $K_{\omega}=k_{\omega} J$ and $K_{q}=k_{q} J$ are constant gain matrices. The gains are multiplied by the inertia matrix so that the gains are proportionally 
higher on the axes with higher moments of inertia, simplifying the gain tuning process such that only two parameters need to be tuned. The angular velocity error is given by $\bar{\omega}_{e}=\bar{\omega}-\bar{\omega}_{d}$, and the quaternion error by:

$$
\bar{q}_{e}=\left[\begin{array}{cccc}
q_{0 d} & q_{1 d} & q_{2 d} & q_{3 d} \\
-q_{1 d} & q_{0 d} & q_{3 d} & -q_{2 d} \\
-q_{2 d} & -q_{3 d} & q_{0 d} & q_{1 d} \\
-q_{3 d} & q_{2 d} & -q_{1 d} & q_{0 d}
\end{array}\right] \bar{q}
$$

where $\bar{\omega}_{d}$ and $\bar{q}_{d}$ are the desired angular velocities and quaternions at time $t$. Only the vector part of the error quaternion $\bar{q}_{e}$ (i.e. $q_{1 e}, q_{2 e}, q_{3 e}$ ) is used in tracking. Note that a gyroscopic term is often added to the proportional-derivative controller of (46). However for axisymmetric and slightly asymmetric spacecraft, and long, slow manoeuvres, this term has negligible effect and so is omitted here [6], [15].

As the natural motion references are not inherently rest-torest, the manoeuvre is planned such that the references bring the spacecraft to the target in time $(T-\tau)$, where $\tau$ is a small period of time. At time $(T-\tau)$ until the end of the manoeuvre at time $T$, a stabilising control is applied to bring the spacecraft to rest at the target. The manoeuvre can be summarised as:

$$
\begin{array}{ll}
\text { For } t=[0, T-\tau) & \bar{\omega}_{d}=\bar{\omega}_{r e f} \\
& \bar{q}_{d}=\bar{q}_{r e f} \\
\text { For } t=[T-\tau, T] & \bar{\omega}_{d}=0 \\
& \bar{q}_{d}=\bar{q}(T)
\end{array}
$$

where the subscript "ref" refers to the natural motion reference tracks generated using parametric optimisation, and $\tau$ is the length of time the stabilising control is applied. In the following simulations, a value of $\tau=20$ seconds is utilised as this was found to be the shortest time required to bring the spacecraft to rest. When quaternion feedback is being considered, $\bar{q}_{d}$ does not vary and is the final desired attitude i.e. $\bar{q}_{d}=\bar{q}(T) \forall t$. Equation (46) then describes a conventional quaternion feedback controller.

We assess the performance of the natural motion tracking in comparison to the benchmark quaternion feedback controller in terms of the accumulated torque [14] of the reaction wheels. That is, the integral of the norm of the control torque vector, given the symbol $I_{N}$. The accumulated torque is used as an indication of the control effort required to carry out the manoeuvre, with lower control effort especially important for small spacecraft due to their limited power and actuation.

For the quaternion feedback and natural motion manoeuvres the gains were adjusted until the final pointing and velocity constraints were satisfied, to 4 decimal places, with the lowest accumulated torque. In the case of the natural motions the gains were tuned using an iterative process. The gains for quaternion feedback were initially tuned using an iterative process and then, to ensure the fairest comparison between the natural motions and quaternion feedback, the quaternion feedback gains were further tuned by using the genetic algorithm function in Matlab. A cost function consisting of the angular velocity and quaternion errors at time $T$ and the total accumulated torque of the manoeuvre was constructed. The optimisation process was repeated until the gains which
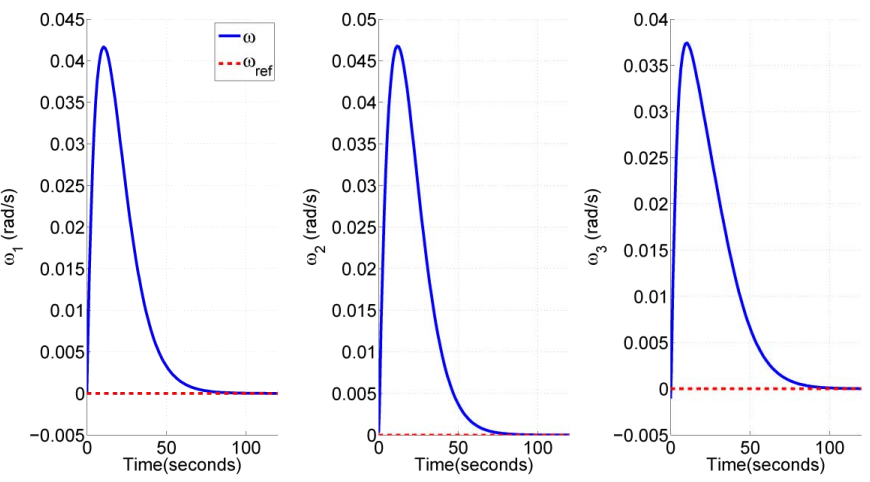

Figure 3: Angular velocities during quaternion feedback manoeuvre for axisymmetric spacecraft from $\bar{q}(0)=$ $\left[\begin{array}{llll}1 & 0 & 0 & 0\end{array}\right]^{T}$ to $\bar{q}(T)=\left[\begin{array}{llll}0.5 & 0.5 & 0.5 & 0.5\end{array}\right]^{T}$.
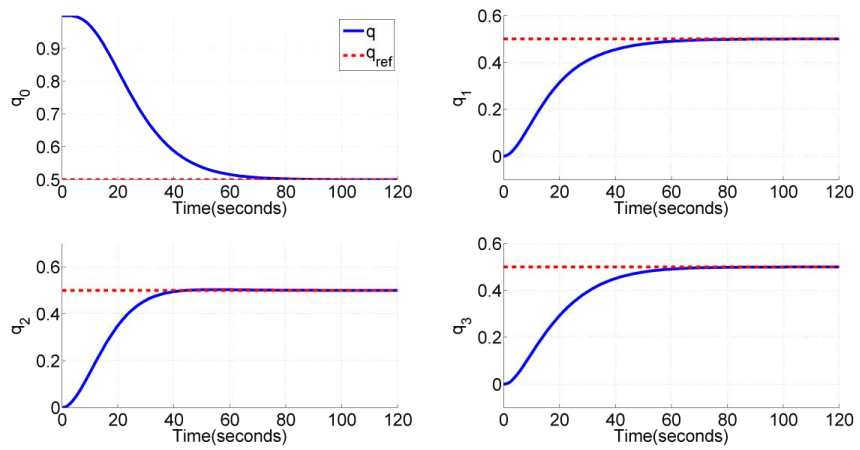

Figure 4: Quaternions during quaternion feedback for axisymmetric spacecraft from $\bar{q}(0)=\left[\begin{array}{llll}1 & 0 & 0 & 0\end{array}\right]^{T}$ to $\bar{q}(T)=$ $\left[\begin{array}{llll}0.5 & 0.5 & 0.5 & 0.5\end{array}\right]^{T}$.

yielded the lowest accumulated torque while matching the desired boundary conditions were found.

A $600 \mathrm{~km}$ altitude circular orbit beginning at the vernal equinox position is utilised in all simulations unless it is stated otherwise.

\section{A. Axisymmetric Case: Example Manoeuvres}

The performance of the axisymmetric natural motions was assessed via comparison with a quaternion feedback benchmark for a spacecraft with principal inertias $J_{1}=0.0109 \mathrm{kgm}^{2}, J_{2}=J_{3}=0.05 \mathrm{kgm}^{2}$. A manoeuvre was carried out from $\bar{q}(0)=\left[\begin{array}{llll}1 & 0 & 0 & 0\end{array}\right]^{T}$ to $\bar{q}(T)=$ $\left[\begin{array}{llll}0.5 & 0.5 & 0.5 & 0.5\end{array}\right]^{T}$ in a time of $T=120$ seconds. The gains used in the simulation were $k_{\omega}=0.2095$ and $k_{q}=0.0222$ for quaternion feedback, and $k_{\omega}=1.81$ and $k_{q}=0.83$ for the natural motions. The results are shown in Figures 3, 4 and 5 for quaternion feedback and Figures 6, 7 and 8 for the natural motion tracking.

It is clear that the natural motion tracking approach is essentially "bang-off-bang" in nature. Initial torques bring the spacecraft angular velocities to those required to perform a natural motion. This is then followed by a "coasting phase" of zero or near zero torque (a small torque is often applied to 
Table III: Comparison between quaternion feedback and natural motions for axisymmetric spacecraft. T=120 seconds.

\begin{tabular}{l|l|l|l|l|l}
\hline \hline Initial quaternion & Final quaternion & $I_{N} Q \cdot F \cdot(\mathrm{Nms})$ & $I_{N}{ }^{N . M(A x i)}(\mathrm{Nms})$ \\
\hline $\bar{q}(0)=\left[\begin{array}{lllllll}1 & 0 & 0 & 0\end{array}\right]^{T}$ & $\bar{q}(T)=\left[\begin{array}{llllll}0.5 & 0.5 & 0.5 & 0.5\end{array}\right]^{T}$ & 0.0068 & 0.0025 \\
$\bar{q}(0)=\left[\begin{array}{llllll}-0.124 & 0.705 & 0.698 & 0.039\end{array}\right]^{T}$ & $\bar{q}(T)=\left[\begin{array}{lllll}-0.768 & 0.557 & -0.0815 & -0.307\end{array}\right]^{T}$ & 0.0103 & 0.0014 \\
$\bar{q}(0)=\left[\begin{array}{llllll}-0.563 & 0.018 & 0.446 & 0.695\end{array}\right]^{T}$ & $\bar{q}(T)=\left[\begin{array}{lllll}-0.596 & 0.65 & 0.371 & 0.29\end{array}\right]^{T}$ & 0.0086 & 0.0013 \\
$\bar{q}(0)=\left[\begin{array}{llllll}-0.202 & -0.811 & -0.151 & 0.528\end{array}\right]^{T}$ & $\bar{q}(T)=\left[\begin{array}{lllll}-0.059 & -0.349 & -0.767 & -0.535\end{array}\right]^{T}$ & 0.0146 & 0.003 \\
$\bar{q}(0)=\left[\begin{array}{llllll}0.588 & 0.749 & 0.098 & -0.289\end{array}\right]^{T}$ & $\bar{q}(T)=\left[\begin{array}{lllll}0.484 & 0.522 & -0.561 & -0.423\end{array}\right]^{T}$ & 0.0046 & 0.0018 \\
\hline \hline
\end{tabular}

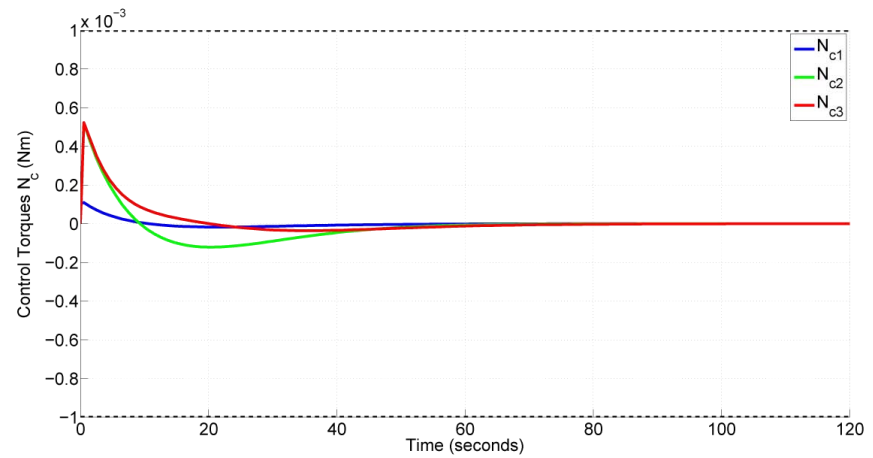

Figure 5: Control torques during quaternion feedback manoeuvre for axisymmetric spacecraft from $\bar{q}(0)=$ $\left[\begin{array}{llll}1 & 0 & 0 & 0\end{array}\right]^{T}$ to $\bar{q}(T)=\left[\begin{array}{llll}0.5 & 0.5 & 0.5 & 0.5\end{array}\right]^{T}$.
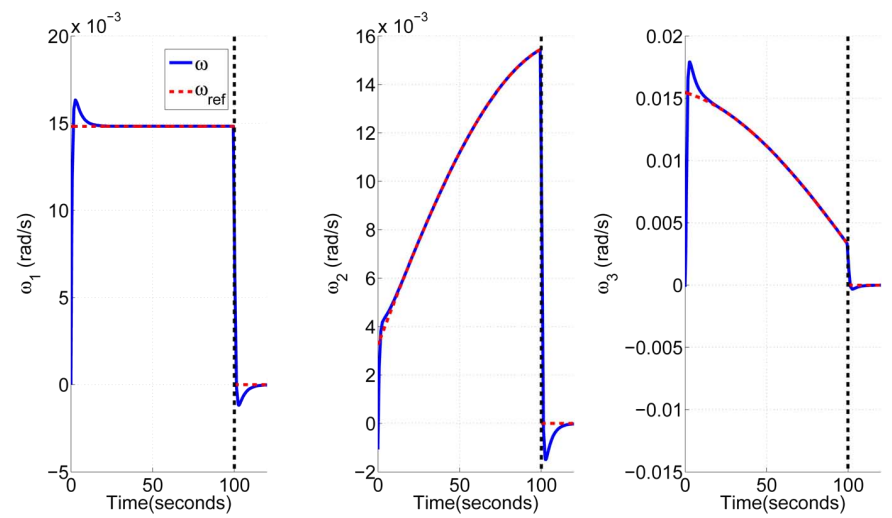

Figure 6: Angular velocities during natural motion manoeuvre for axisymmetric spacecraft from $\bar{q}(0)=\left[\begin{array}{llll}1 & 0 & 0 & 0\end{array}\right]^{T}$ to $\bar{q}(T)=\left[\begin{array}{llll}0.5 & 0.5 & 0.5 & 0.5\end{array}\right]^{T}$. Vertical line indicates end of natural motion tracking and switch to stabilising control.

compensate for disturbances) as the spacecraft moves toward the target attitude. When the target attitude is achieved at time $(T-\tau)$, the angular velocity references are then switched to stabilise the spacecraft at the target. This results in a final torquing phase to bring the spacecraft to rest at the target, the start of which is indicated by a dotted vertical line in the figures. In contrast we note that the quaternion feedback controller, which tracks a constant rather than time varying reference, results in the quaternions following broadly sigmoidal paths during the manoeuvre.

Table III summarises the results of this and several other manoeuvres. The superscripts "Q.F." and "N.M(Axi)" refer to quaternion feedback and the axisymmetric natural motion
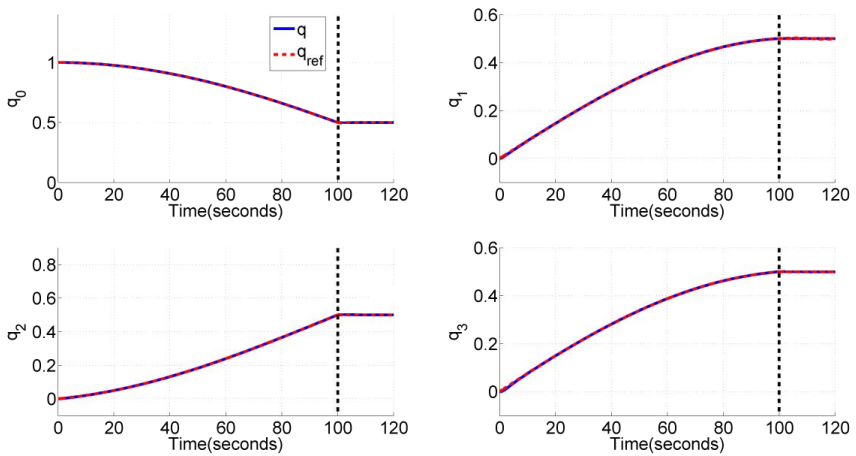

Figure 7: Quaternions during natural motion manoeuvre for axisymmetric spacecraft from $\bar{q}(0)=\left[\begin{array}{llll}1 & 0 & 0 & 0\end{array}\right]^{T}$ to $\bar{q}(T)=\left[\begin{array}{llll}0.5 & 0.5 & 0.5 & 0.5\end{array}\right]^{T}$. Vertical line indicates end of natural motion tracking and switch to stabilising control.

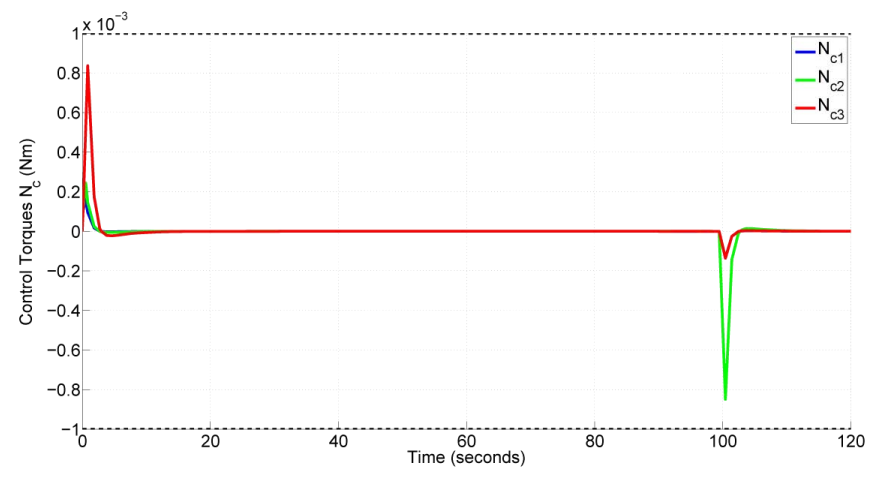

Figure 8: Control torques during natural motion manoeuvre for axisymmetric spacecraft from $\bar{q}(0)=\left[\begin{array}{llll}1 & 0 & 0 & 0\end{array}\right]^{T}$ to $\bar{q}(T)=\left[\begin{array}{llll}0.5 & 0.5 & 0.5 & 0.5\end{array}\right]^{T}$.

manoeuvres respectively.

The natural motion tracking offers significant savings in accumulated torque over the more computationally efficient quaternion feedback for a range of manoeuvres. However the peak torques of the natural motion method are generally slightly higher than those for quaternion feedback.

\section{B. Asymmetric Case: Example Manoeuvres}

The motion planner for asymmetric spacecraft was then tested in simulation for a spacecraft with principal inertias $J_{1}=0.0109 \mathrm{kgm}^{2}, J_{2}=0.0504 \mathrm{kgm}^{2}$ and $J_{3}=0.0506 \mathrm{kgm}^{2}$. A manoeuvre from $\bar{q}(0)=\left[\begin{array}{llll}0.208 & 0.622 & 0.431 & 0.620\end{array}\right]^{T}$ to $\bar{q}(T)=\left[\begin{array}{llll}1 & 0 & 0 & 0\end{array}\right]^{T}$ in a time of $T=120$ seconds is illustrated. The gains used were $k_{\omega}=0.194$ and $k_{q}=0.01972$ 
Table IV: Comparison between quaternion feedback and natural motions for slightly asymmetric spacecraft. T=120 seconds.

\begin{tabular}{|c|c|c|c|}
\hline Initial quaternion & Final quaternion & 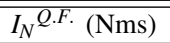 & $I_{N}^{N . M(A s y)}(\mathrm{Nms})$ \\
\hline $\bar{q}(0)=\left[\begin{array}{llll}0.208 & 0.622 & 0.431 & 0.620\end{array}\right]^{T}$ & $\bar{q}(T)=\left[\begin{array}{llll}1 & 0 & 0 & 0\end{array}\right]^{T}$ & 0.008 & 0.0024 \\
\hline $\bar{q}(0)=\left[\begin{array}{llll}0.049 & -0.77 & -0.547 & 0.32\end{array}\right]^{T}$ & $\bar{q}(T)=\left[\begin{array}{llll}0.64 & -0.68 & -0.34 & -0.13\end{array}\right]^{T}$ & 0.0083 & 0.0016 \\
\hline $\bar{q}(0)=\left[\begin{array}{llll}-0.12 & 0.76 & -0.63 & 0.068\end{array}\right]^{T}$ & $-0.15 \quad-0.83 \quad 0.32]^{T}$ & 0.0076 & 0.0033 \\
\hline $\bar{q}(0)=\left[\begin{array}{ll}-0.32 & 0.92\end{array}\right.$ & $\bar{q}(T)=\left[\begin{array}{llll}0.39 & 0.62 & -0.62 & 0.27\end{array}\right]^{T}$ & 0.0115 & 0.0022 \\
\hline $\bar{q}(0)=\left[\begin{array}{llll}0.45 & 0.26 & -0.38 & 0.76\end{array}\right]^{T}$ & $\bar{q}(T)=\left[\begin{array}{llll}-0.65 & -0.4 & -0.43 & -0.49\end{array}\right]^{T}$ & 0.0119 & 0.0043 \\
\hline
\end{tabular}
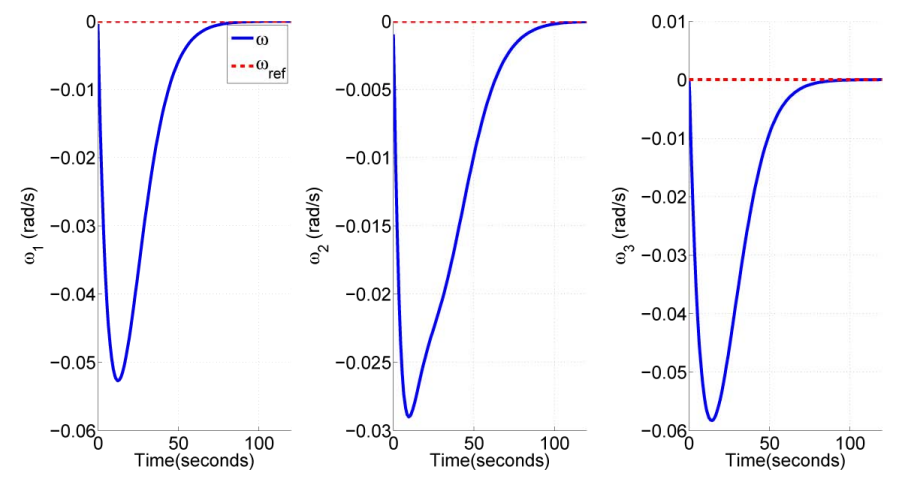

Figure 9: Angular velocities during quaternion feedback manoeuvre for asymmetric spacecraft from $\bar{q}(0)=\left[\begin{array}{llll}0.208 & 0.622 & 0.431 & 0.620\end{array}\right]^{T}$ to $\bar{q}(T)=\left[\begin{array}{llll}1 & 0 & 0 & 0\end{array}\right]^{T}$.
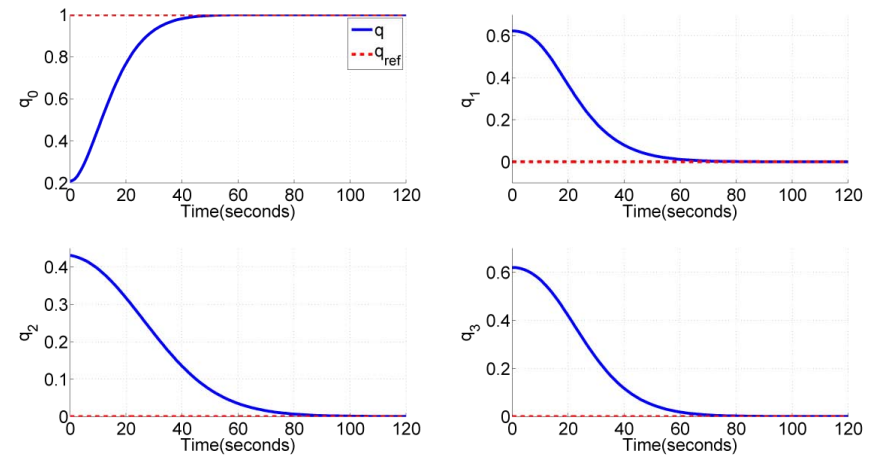

Figure 10: Quaternions during quaternion feedback manoeuvre for asymmetric spacecraft from $\bar{q}(0)=\left[\begin{array}{llll}0.208 & 0.622 & 0.431 & 0.620\end{array}\right]^{T}$ to $\bar{q}(T)=\left[\begin{array}{llll}1 & 0 & 0 & 0\end{array}\right]^{T}$.

for quaternion feedback, and $k_{\omega}=1.8$ and $k_{q}=0.86$ for the natural motions. The results are shown in Figures 9, 10 and 11 for quaternion feedback and Figures 12, 13 and 14 for the natural motion tracking.

Table IV summarises the results of this and several other manoeuvres. The superscript "N.M(Asy)" refers to the asymmetric natural motion manoeuvre.

It is clear that the natural motion tracking in the asymmetric case once again offers significant savings in accumulated torque over quaternion feedback alone.

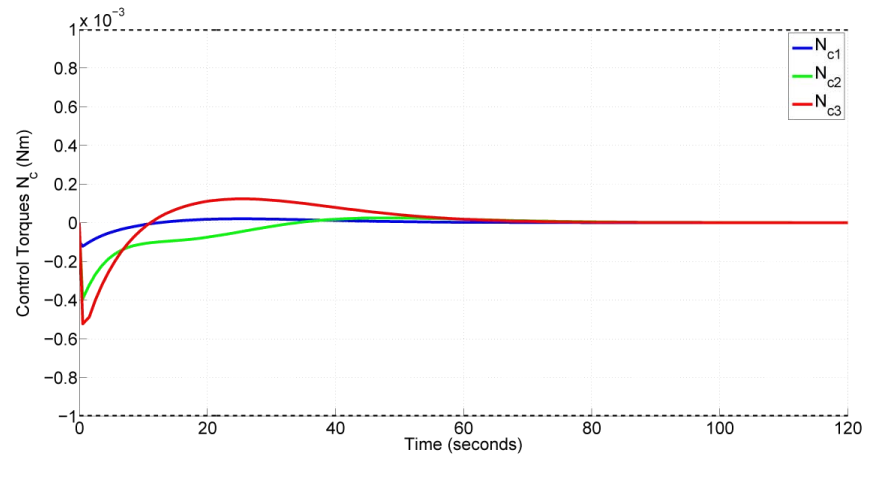

Figure 11: Control torques during quaternion feedback manoeuvre for asymmetric spacecraft from $\bar{q}(0)=\left[\begin{array}{llll}0.208 & 0.622 & 0.431 & 0.620\end{array}\right]^{T}$ to $\bar{q}(T)=\left[\begin{array}{llll}1 & 0 & 0 & 0\end{array}\right]^{T}$.
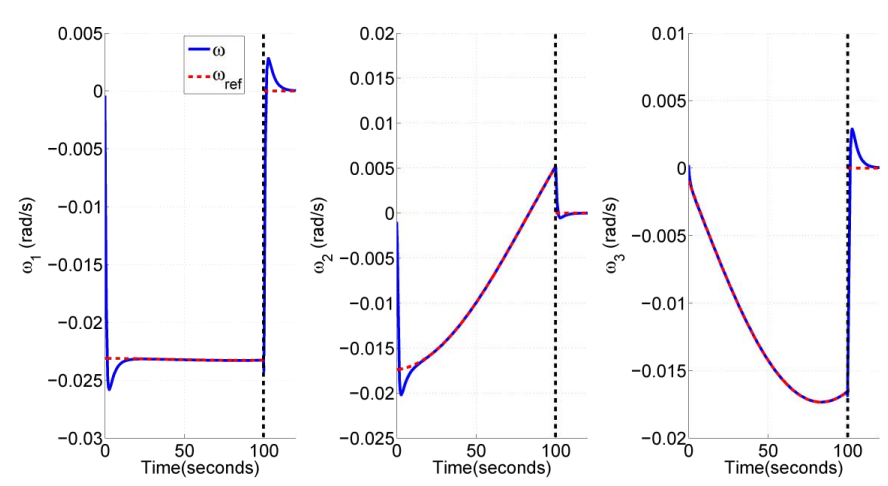

Figure 12: Angular velocities during natural motion manoeuvre for asymmetric spacecraft from $\bar{q}(0)=\left[\begin{array}{llll}0.208 & 0.622 & 0.431 & 0.620\end{array}\right]^{T}$ to $\bar{q}(T)=\left[\begin{array}{llll}1 & 0 & 0 & 0\end{array}\right]^{T}$. Vertical line indicates end of natural motion tracking and switch to stabilising control.

\section{Effect of Inertia}

While ideally the asymmetric motion planner would be used to plan motions for asymmetric spacecraft, the elliptic functions present in this motion planner result in increased computation times in comparison with the axisymmetric motion planner. This will be discussed in detail in Section VI. However, as most Cubesat spacecraft are only slightly asymmetric, it may be possible to use the axisymmetric motion planner in these cases.

In this section we assess whether the axisymmetric motion planning method can still offer savings in accumulated torque even as the inertias vary from those of the axisymmetric 

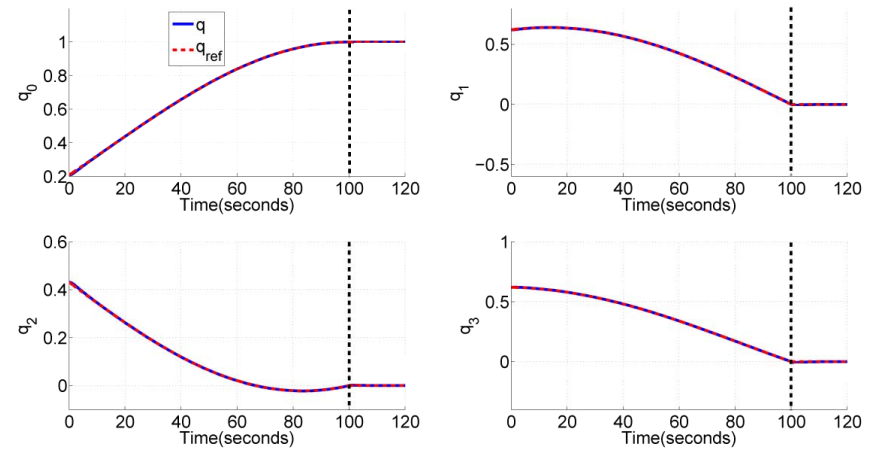

Figure 13: Quaternions during natural motion manoeuvre for asymmetric spacecraft from $\bar{q}(0)=\left[\begin{array}{llll}0.208 & 0.622 & 0.431 & 0.620\end{array}\right]^{T}$ to $\bar{q}(T)=\left[\begin{array}{llll}1 & 0 & 0 & 0\end{array}\right]^{T}$. Vertical line indicates end of natural motion tracking and switch to stabilising control.

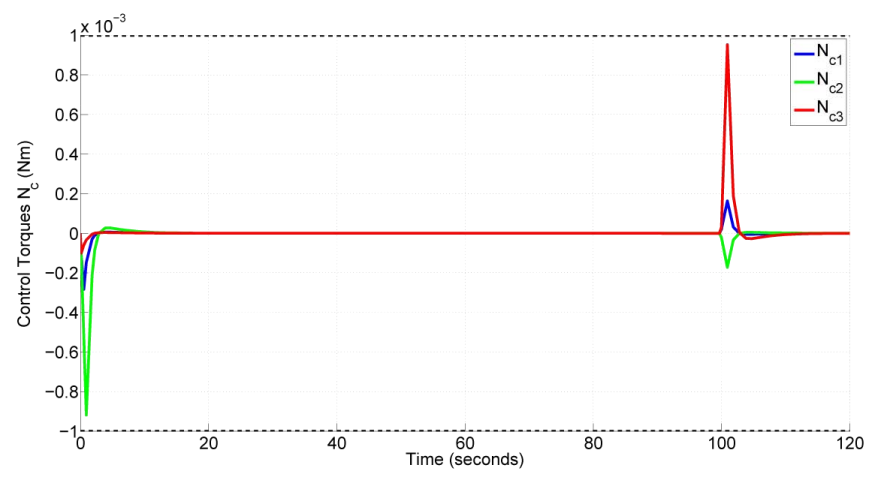

Figure 14: Control torques during natural motion manoeuvre for asymmetric spacecraft from $\bar{q}(0)=\left[\begin{array}{llll}0.208 & 0.622 & 0.431 & 0.620\end{array}\right]^{T}$ to $\bar{q}(T)=\left[\begin{array}{llll}1 & 0 & 0 & 0\end{array}\right]^{T}$.

spacecraft. This also serves as a study of how sensitive the method is to the inertia fluctuations which could arise during a mission (e.g. due to the deployment of solar panels or the jettison of a payload).

The references generated in Section V-A for a 120second manoeuvre from $\bar{q}(0)=\left[\begin{array}{llll}1 & 0 & 0 & 0\end{array}\right]^{T}$ to $\bar{q}(T)=\left[\begin{array}{llll}0.5 & 0.5 & 0.5 & 0.5\end{array}\right]^{T}$ for the axisymmetric spacecraft were applied to several asymmetric spacecraft with inertias ranging from $\pm 0-100 \%$ difference over the inertias of the axisymmetric spacecraft. In order to determine the effect of the inertia error on the axisymmetric natural motions, references were also generated for each spacecraft configuration using the asymmetric motion planner, and a quaternion feedback benchmark again included for comparison. The results are shown in Table V.

It is obvious that the accumulated torque required to track the axisymmetric references increases as the inertia uncertainty increases. However for a slightly asymmetric spacecraft, the axisymmetric references require only slightly more torque to track. Therefore in these cases the less computationally intensive axisymmetric motion planner would be the more practical choice. It should also be noted that the natural motion references for the axisymmetric spacecraft outperform quaternion feedback in terms of accumulated torque, even in the presence of considerable inertia error.

\section{Effect of Manoeuvre Time}

In this section we investigate the effect of manoeuvre time on the natural motion method. For a manoeuvre from $\bar{q}(0)=\left[\begin{array}{llll}1 & 0 & 0 & 0\end{array}\right]^{T}$ to $\bar{q}(T)=\left[\begin{array}{llll}0.5 & 0.5 & 0.5 & 0.5\end{array}\right]^{T}$, the time is varied from the shortest time quaternion feedback slew which could be performed without momentum saturation of the reaction wheels, 50seconds, up to a manoeuvre time of 420seconds. The results are shown in Table VI.

Table VI: Comparison between quaternion feedback and natural motions with varying manoeuvre time for axisymmetric spacecraft manoeuvre from $\bar{q}(0)=\left[\begin{array}{llll}1 & 0 & 0 & 0\end{array}\right]^{T}$ to $\bar{q}(T)=$ $\left[\begin{array}{llll}0.5 & 0.5 & 0.5 & 0.5\end{array}\right]^{T}$

\begin{tabular}{l|l|l|l}
\hline \hline T (seconds) & $I_{N}{ }^{Q . F .}(\mathrm{Nms})$ & $I_{N}{ }^{N . M(A x i)}(\mathrm{Nms})$ & $\%$ Saving \\
\hline 50 & 0.0148 & 0.0083 & 44 \\
120 & 0.0068 & 0.0022 & 68 \\
220 & 0.0061 & 0.0011 & 82 \\
420 & 0.0043 & 0.00057 & 87 \\
\hline \hline
\end{tabular}

The $\%$ saving is defined as $\left(I_{N} Q . F .-I_{N}^{N . M(A s y)}\right) / I_{N} Q . F . \times$ 100. It can be seen that the natural motion method is still advantageous throughout, though the savings in accumulated torque are greater for greater time periods. For shorter time periods the stabilising control discussed in Section $\mathrm{V}$ takes up a greater proportion of the total manoeuvre time. In other words, as manoeuvre time decreases the length of time which the natural motion references are tracked decreases and the manoeuvres become increasingly similar to conventional quaternion feedback manoeuvres. This accounts for the decrease in \% saving for shorter time periods.

\section{E. Effect of Orbital Altitude, Position and Inclination}

Next, the effect of the spacecraft's orbit on the natural motion method was investigated. Disturbance torques such as air drag, solar radiation pressure and gravity gradient are dependent on orbit altitude, while the residual dipole torques are more significant at the poles due to the increased magnetic field strength. As the natural motion method is based around the motion of a rigid body in a disturbance free environment, the method may not be suitable for manoeuvres in a high disturbance environment and so it is important to test the method in these cases.

For an axisymmetric spacecraft on a $300 \mathrm{~km}$ altitude orbit beginning at the vernal equinox, a manoeuvre from $\bar{q}(0)=$ $\left[\begin{array}{llll}1 & 0 & 0 & 0\end{array}\right]^{T}$ to $\bar{q}(T)=\left[\begin{array}{llll}0.5 & 0.5 & 0.5 & 0.5\end{array}\right]^{T}$ results in accumulated torques of $I_{N}=0.0024 \mathrm{Nms}$ for the natural motions. This is slightly higher than the $I_{N}=0.0022 \mathrm{Nms}$ obtained for the manoeuvre at $600 \mathrm{~km}$. Therefore in this case the natural motion method is not significantly influenced by the increased air drag and gravity gradient disturbance torques. Testing at a $900 \mathrm{~km}$ altitude yielded the same results as for a $600 \mathrm{~km}$ altitude, suggesting that the decrease in air drag and gravity 
Table V: Effect of varying spacecraft inertia on tracking of axisymmetric references from $\bar{q}(0)=\left[\begin{array}{llll}1 & 0 & 0 & 0\end{array}\right]^{T}$ to $\bar{q}(T)=$ $\left[\begin{array}{llll}0.5 & 0.5 & 0.5 & 0.5\end{array}\right]^{T}$ in 120 seconds

\begin{tabular}{l|l|l|l}
\hline \hline Principal Inertias $\left(\mathrm{kgm}^{2}\right)$ & $I_{N}$ N.M(Axi) $(\mathrm{Nms})$ & $I_{N}{ }^{N . M(A s y)}(\mathrm{Nms})$ & $I_{N}$ Q.F. $(\mathrm{Nms})$ \\
\hline$J_{1}=0.0109 \mathrm{kgm}^{2}, J_{2}=0.0504 \mathrm{kgm}^{2}, J_{3}=0.0506 \mathrm{kgm}^{2}$ & 0.0023 & 0.0022 & 0.0069 \\
$J_{1}=0.0164 \mathrm{kgm}^{2}, J_{2}=0.075 \mathrm{kgm}^{2}, J_{3}=0.025 \mathrm{kgm}^{2}$ & 0.0033 & 0.0018 & 0.0106 \\
$J_{1}=0.0218 \mathrm{kgm}^{2}, J_{2}=0.1 \mathrm{kgm}^{2}, J_{3}=0.05 \mathrm{kgm}^{2}$ & 0.0047 & 0.003 & 0.0123 \\
\hline \hline
\end{tabular}

gradient torques and the increase in solar radiation pressure torques at higher altitudes has little effect on the manoeuvres. Additionally, beginning the manoeuvre at the poles on a $600 \mathrm{~km}$ altitude orbit rather than at the vernal equinox results in a small increase in the required accumulated torques, with $I_{N}=0.0023 \mathrm{Nm}$ for the natural motions.

These results show that the variation in disturbance torques caused by varying the orbital parameters has little effect on the accumulated torque of the natural motion method. Therefore it may be possible to effectively switch the control off during the "coasting phase": that is, the section of the manoeuvre between the two main torquing phases. For altitudes less than $600 \mathrm{~km}$ this proves infeasible as the increased air drag torque results in a significant drift from the reference tracks if no control is applied. However for the $600 \mathrm{~km}$ altitude manoeuvre beginning at the vernal equinox described above we find that the control torques during the "coasting phase" can be switched off once the norm of the error between the actual and desired values of angular velocities is less than $1 \times 10^{-4} \mathrm{rad} / \mathrm{s}$, and back on when the error was greater than this value, with no impact on the accumulated torque required or the accuracy of the manoeuvre. In contrast for the manoeuvre near the poles - a slightly higher disturbance environment - applying the same method of switching the control off when $\left\|\bar{\omega}_{e}\right\|<1 \times 10^{-4} \mathrm{rad} / \mathrm{s}$, we find that the accuracy of the manoeuvre suffers slightly when the control is switched off during the coasting phase, with a final attitude of $\bar{q}(T)=\left[\begin{array}{llll}0.4999 & 0.5001 & 0.5001 & 0.4998\end{array}\right]^{T}$ achieved. This is as a result of the increased magnetic dipole torque, which means that the drift from the reference tracks is too great to compensate for when the control is switched back on. However this strategy may still be feasible if a high pointing accuracy is not required.

Therefore, as expected, the natural motions require slightly more accumulated torque to track in lower Earth orbits due to the increased disturbance torques. In contrast in low disturbance environments the control can be switched off during the "coasting phase", resulting in potential savings in computation and power. In these cases the 3-axes natural attitude motions are roughly analogous to the single-axis "bang-off-bang" controls augmented by linear feedback proposed by Sidi [4], in that the control is only switched on near the boundaries of the motion to first achieve the desired angular velocities and then to stabilise the spacecraft at the target attitude.

\section{F. Constrained Repointing}

We now illustrate the method of probing the set of natural motions which satisfy the boundary conditions on the target attitude to generate a constrained repointing manoeuvre.

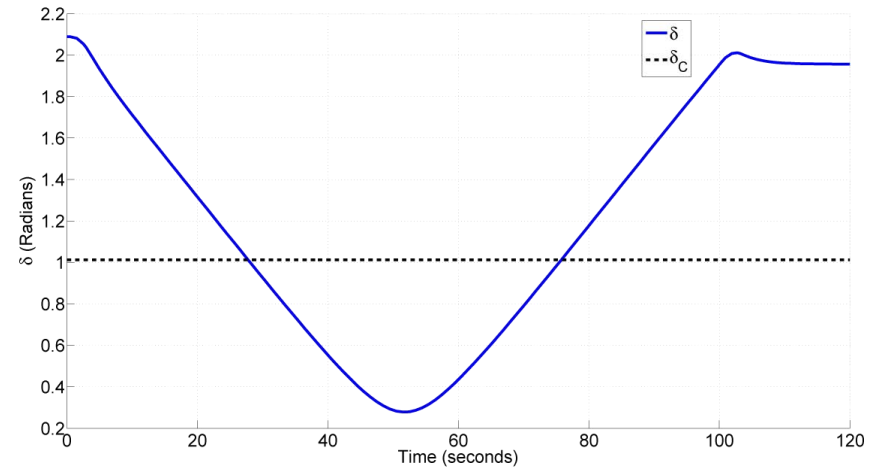

Figure 15: Angle between body $\mathrm{x}$-axis and cone centreline unit vectors for manoeuvre from $\bar{q}(0)=\left[\begin{array}{llll}1 & 0 & 0 & 0\end{array}\right]^{T}$ to $\bar{q}(T)=\left[\begin{array}{llll}-0.449 & -0.23 & 0.566 & 0.65\end{array}\right]^{T}$. Constraint is violated.

We consider a 120second manoeuvre from $\bar{q}(0)=$ $\left[\begin{array}{llll}1 & 0 & 0 & 0\end{array}\right]^{T}$ to $\bar{q}(T)=\left[\begin{array}{llll}-0.449 & -0.23 & 0.566 & 0.65\end{array}\right]^{T}$ for the axisymmetric spacecraft from Section V-A, with a star tracker mounted in the direction of the body $\mathrm{x}$-axis unit vector, $n_{p}=\left[\begin{array}{lll}1 & 0 & 0\end{array}\right]^{T}$. The centre of the forbidden cone is specified as $n_{c}=\left[\begin{array}{lll}-0.495 & 0.81 & 0.317\end{array}\right]^{T}$ and the cone half angle as $\delta_{c}=58^{\circ}$. This corresponds to the Moon cone as defined by Mengali [17]. An initial optimisation to minimise the cost function (43) yields a solution which violates this constraint, as shown in Figure 15.

Now, incrementing the angular velocities $\omega_{1}{ }^{*}(0), \omega_{2}{ }^{*}(0)$ and $\omega_{3}{ }^{*}(0)$ by $p_{1}, p_{2}$ and $p_{3}$ respectively and performing a further parametric optimisation to minimise the cost function (45) leads to a manoeuvre which does not violate the constraint, as shown in Figure 16. It is evident that the constraint $\delta_{p}>\delta_{c} \forall t$ is respected and the camera would not pass through the forbidden zone during the manoeuvre. Therefore the natural motion method has been used to generate a constrained slew.

\section{Computational Suitability}

In order for a control or guidance method to be suitable for implementation on-board a nano-spacecraft, the method must be both low torque and computationally efficient. As the natural motion planning method has proven to require significantly less accumulated torque than a quaternion feedback benchmark for a wide range of manoeuvres, the suitability of the method for on-board implementation will now be discussed.

The references were generated in the programme Mathematica, using a $2 \mathrm{GHz}$, dual-core PC. While this is considerably more powerful than the $30 \mathrm{MHz}$ processor on-board UKube-1, the 


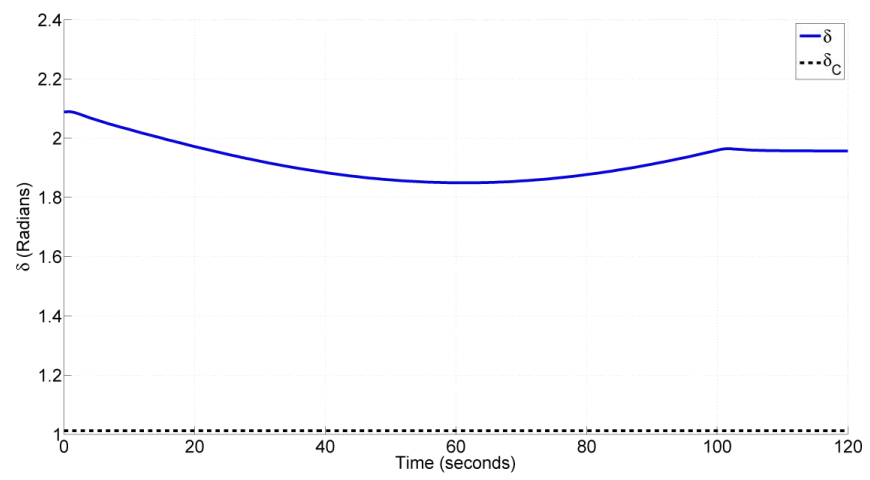

Figure 16: Angle between body $\mathrm{x}$-axis and cone centreline unit vectors for manoeuvre from $\bar{q}(0)=\left[\begin{array}{llll}1 & 0 & 0 & 0\end{array}\right]^{T}$ to $\bar{q}(T)=\left[\begin{array}{llll}-0.449 & -0.23 & 0.566 & 0.65\end{array}\right]^{T} . \delta_{p}>\delta_{c} \forall t$.

difference is less marked for other $3 \mathrm{U}$ Cubesats such as OPSSAT which has an $800 \mathrm{MHz}$ dual-core processor. Using the solver "DifferentialEvolution", which as stated previously uses a direct search method to find the globally optimal solution, the axisymmetric references take, on average, 0.17 seconds to generate. The asymmetric references, however, take between 15 and 20 minutes to generate using the same software. This is due to the presence of the incomplete Jacobi elliptic integral of the third kind in the analytic expressions for the quaternions (33). While mathematically elegant, current computer software finds this Jacobi elliptic integral computationally heavy. As a result, the asymmetric references, despite offering significant fuel savings, are not necessarily any more efficient than using a pseudospectral optimisation method such as PSOpt ${ }^{4}$ or a multiple shooting method to produce reference motions. Therefore the asymmetric motion planner is not presently suitable for on-board implementation.

In contrast the generation of the axisymmetric references requires significantly less computation and as shown in Section V-C these references can be applied to axisymmetric and near axisymmetric spacecraft. However, the method of finding the globally optimal angular velocities via a parametric optimisation means that the axisymmetric natural motions are less computationally efficient than quaternion feedback, where only the final quaternion is tracked. As noted previously, the references are essentially "bang-off-bang" in nature and so provide significant savings in control effort. Furthermore, in low disturbance environments the control can be switched off during the region of low or zero torque named the "coasting phase", potentially providing savings in power and computation. As a consequence, the reduction in control effort offered by the axisymmetric natural motion method on small spacecraft has to be weighed against the computation required to initially generate the references. While quantifying this tradeoff will be the subject of future research, we proceed with a discussion of the relative strengths and weaknesses of another guidance method in artificial potential functions (APFs).

\footnotetext{
${ }^{4}$ http://www.psopt.org/
}

\section{A. Trade-off Between Computation and Control Effort}

While the reliance on quaternion feedback and eigenaxis controllers for attitude manoeuvres make the generation of time-dependent references for attitude control relatively rare, the use of artificial potential functions [16] has been proposed as a non-optimal guidance method. Artificial potential functions require less computation than the natural motion method as they do not require parametric optimisation; rather they make use of Lyapunov functions to assign an attractive potential to the target orientation, and generate the desired references using inverse methods [16]. Artificial potential functions also provide a simple and effective means of obstacle avoidance. However in contrast to the natural motion method, the APF method is not inherently low torque and can demand excessive control effort if care is not taken to constrain slew rates and maximum applied torques [33], [34]. In addition, as the manoeuvres are not based around the natural motion of the free rigid body, the references must be tracked throughout and so the artificial potential function method cannot be utilised in a quasi-open loop manner to save on power and computation. Thus we see that the artificial potential functions, in contrast to the natural motion method, require less computation at the expense of a likely increase in control effort. A thorough comparison with the natural motion planning method will be the subject of future work in order to assess this trade-off between computational intensity and control effort.

\section{B. On-board Implementation}

The analytical approach presented in this paper offers the potential to reduce the torque requirement with respect to using quaternion feedback alone, at the expense of an increase in computation. However, although the motion planning problem is reduced to one of parametric optimisation, further work will be required to assess the most appropriate parametric optimisation method for on-board implementation. We proceed with a brief discussion of steps which could be taken to make the natural motion method easier to implement on-board a nano-spacecraft.

As described above, the references were generated on a $2 \mathrm{GHz}$, dual-core computer using a global parametric optimisation method. However, it may not be feasible to implement a global parametric optimisation method on-board a nano-spacecraft with a processor in the $30-800 \mathrm{MHz}$ range. In this case, it may be possible to implement a simple gradient based search method [35] instead. In these methods the search is proportional to the negative of the gradient of the cost function, with the aim of finding a local rather than a global minimum. These methods are computationally more efficient than the direct search methods, at the expense of only finding a local minimum.

A comparison of the local gradient based inbuilt Mathematica method "FindMinimum" with the global direct search method "DifferentialEvolution" for 50 randomly generated manoeuvres found that the performance of the "FindMinimum" method was highly dependent on the given initial guess. For example when the solver was given an initial guess of $\bar{\omega}(0)=\left[\begin{array}{lll}0.1 & 0.1 & 0.1\end{array}\right]^{T} \times 10^{-3} \mathrm{rad} / \mathrm{s}$, 
the gradient based method gave the same results as the more computationally intensive global method "DifferentialEvolution" in approximately $60 \%$ of cases. In the remaining $40 \%$ of cases, manual tuning of the initial guess resulted in the solvers achieving the same results. Therefore while the "FindMinimum" method may require less computation, it is heavily reliant on heuristics in the form of a good initial guess in order to function effectively. The natural motion references only require the optimal initial angular velocities as inputs. Therefore it may be possible to store the analytical equations for the natural motions onboard the spacecraft, but to perform the necessary parametric optimisation at the ground station. This would eliminate the need to perform the parametric optimisation on-board the spacecraft and would only necessitate the uploading of the optimal initial angular velocity vector. A problem with this approach is that if manoeuvres could be generated on a powerful computer at a ground station, more computationally intensive methods such as PSOpt could be used instead to generate potentially torque optimal references. However in these cases it would be necessary to upload the time history of the entire manoeuvre rather than just a simple vector, which would require significantly more on-board memory.

It is clear that while planning attitude manoeuvres around the natural motions of a rigid body is beneficial in terms of control effort in comparison to a quaternion feedback benchmark, the reliance on global parametric optimisation methods at present means that more work is required to assess the performance of the method when faced with constraints on processing power, and to determine the most practical reference generation method for on-board implementation.

\section{CONCLUSIONS}

The derived analytical equations of motion for axisymmetric and asymmetric spacecraft can be utilised to produce natural attitude motions by parametric optimisation. The natural motion planning method offers significant savings in accumulated torque over a quaternion feedback benchmark, and the manoeuvres are essentially "bang-off-bang" in nature. The method is robust to errors in inertia and to the effects of disturbances. Indeed, in low disturbance environments the control can be switched off during a "coasting phase" to save on computation, giving rise to quasi-open loop 3-axis "bangoff-bang" manoeuvres. The set of natural motions which match the final attitude can also be probed to produce constrained slews.

The generation of references in the asymmetric case does not offer an advantage over numerical optimisation for onboard use due to the difficulty in evaluating the Jacobi elliptic integral, which makes the method computationally heavy. The axisymmetric references, however, require less computation to generate and may be applicable to axisymmetric and near axisymmetric spacecraft such as UKube-1 and OPS-SAT. However the natural motion method requires a parametric optimisation to generate the references, and so further work is required to determine the most suitable method of implementing the method on-board a resource limited nano-spacecraft.
To address these issues, future work will see the method tested on an attitude control testbed currently under construction at the University of Strathclyde. The natural motion planning method will be implemented on a $30 \mathrm{MHz}$ processor, which will enable the performance of the method in the face of reduced computation, errors due to inaccurate sensing and actuator constraints to be assessed. Allied to this will be finding a means to improve the computational efficiency of the asymmetric method, as at present the savings in accumulated torque are greatly outweighed by the time taken to generate the references due to the presence of an incomplete Jacobi elliptic integral of the third kind.

Additionally, as the quaternion feedback controller causes the quaternions to evolve in broadly sigmoidal curves, the possibility of generating and tracking sigmoidal, rather than constant, quaternion references between the initial and final attitudes will be investigated to determine if this results in a saving in control effort when compared to the natural motion method. Finally, the natural motion method will be compared to those obtained via non-linear optimal control, to assess how close to optimal the natural motions are, and to other non-optimal guidance methods such as the artificial potential function method. The aim of this comparison will be to determine whether the savings in accumulated torque offered by the natural motion method justify the increased computation required to generate the references.

\section{REFERENCES}

[1] Barba, P.M. and Aubrun, J.N., "Satellite Attitude Acquisition by Momentum Transfer", AIAA Journal Vol. 14, pp. 1382-1386, 1976.

[2] Vadali, S.R. and Junkins, J.L., "Spacecraft Large Angle Rotational Maneuvers with Optimal Momentum Transfer", Journal of Astronautical Sciences, Vol. XXXI, No.2, pp. 217-235, 1983.

[3] Raus, R., Gao, Y., Wu, Y. and Watt, M., "Analysis of State-of-theArt Single-Thruster Attitude Techniques for Spinning Penetrator", Acta Astronautica, Vol. 76, pp. 60-78, 2012.

[4] Sidi, M.J., "Space Dynamics and Control: A Practical Engineering Approach", Cambridge University Press, 7, pp. 197-199, 1997.

[5] D'Amario, L.A. and Stubbs, G.S., "A New Single-Rotation-Axis Autopilot for Rapid Spacecraft Attitude Maneuvers", Journal of Guidance and Control, Vol. 2, No. 4, pp. 339-346, 1979.

[6] Wie, B., Weiss, H., and Arapostathis, A., "Quaternion feedback regulator for spacecraft eigenaxis rotations", Journal of Guidance, Control, and Dynamics, Vol. 12, No. 3, pp. 375-380, 1989.

[7] Steyn, W.H., "Near-Minimum-Time Eigenaxis Rotation Maneuvers Using Reaction Wheels", Journal of Guidance, Control, and Dynamics, Vol. 18, No. 5, pp. 1184-1189, 1995.

[8] Wie, B., Bailey, D. and Heiberg, C., "Rapid Multitarget Acquisition and Pointing Control of Agile Spacecraft", Journal of Guidance, Control, and Dynamics, Vol. 25, No. 1, pp. 96-104, 2002.

[9] Verbin, D., Lappas, V.J. and Ben-Asher, J.Z., "Time-Efficient Angular Steering Laws for Rigid Satellites", Journal of Guidance, Control, and Dynamics, Vol. 34, No. 3, pp. 878-892, 2011.

[10] Lawton, J., Beard, R., and Mclain, T., "Successive Galerkin approximation of nonlinear optimal attitude control", Proceedings of the American Control Conference, San Diego, CA, June 1999.

[11] Wie, B., Barba, P.M., "Quaternion Feedback for Spacecraft Large Angle Maneuvers", Journal of Guidance, Control, and Dynamics, Vol. 8, No. 3, pp. 360-365, 1985.

[12] Vadali, S. R. and Junkins, J. L., "Optimal Open-Loop and Stable Feedback Control of Rigid Spacecraft Attitude Maneuvers", Journal of the Astronautical Sciences, Vol. 32, pp. 105-122, 1984.

[13] Krstić, M. and Tsiotras, P., "Inverse optimal stabilization of a rigid spacecraft", IEEE Transactions on Automatic Control, Vol. 44, No. 5, pp. 1042-1049, 1999.

[14] Horri, N. M., Palmer, P. and Roberts, M., "Energy Optimal Spacecraft Attitude Control Subject to Convergence Rate Constraints", Control Engineering Practice, Vol. 19, pp. 1297-1314, 2011. 
[15] Horri, N. M., Palmer, P. and Roberts, M., "Gain-Scheduled Inverse Optimal Satellite Attitude Control", IEEE Transactions on Aerospace and Electronic Systems, Vol. 48, No. 3, pp. 2437-2457, 2012.

[16] McInnes, C. R., "Non-linear Control for Large Angle Attitude Slew Manoeuvres", Proceedings Third International Conference on Spacecraft Guidance, Navigation and Control Systems, ESTEC, Noordwijk, the Netherlands, 1997.

[17] Mengali, G. and Quarta, A.A., "Spacecraft Control with Constrained Fast Reorientation and Accurate Pointing", The Aeronautical Journal, Vol. 108, No.1080, pp. 85-91, 2004.

[18] Maclean, C., Pagnozzi, D. and Biggs, J.D., "Computationally Light Attitude Controls for Resource Limited Nano-spacecraft", In: Proceedings of the 2011 International Astronautical Congress, October 2011, Cape Town, South Africa.

[19] Pagnozzi, D., Maclean, C. and Biggs, J.D., "A New Approach to the Solution of Free Rigid Body Motion for Attitude Maneuvers", In: European Control Conference, July 2013, Zurich, Switzerland.

[20] Wie, B., "Space Vehicle Dynamics and Control," AIAA Education Series, 2nd Edition, pp. 345, pp. 387, pp. 364-365, 2008.

[21] Whittaker, E. T., "A Treatise on the Analytical Dynamics of Particles and Rigid Bodies", pp. 159-161, Cambridge Mathematical Library, Cambridge University Press, 1999.

[22] Bates, L. and Fassò, F., "The Conjugate Locus for the Euler Top I. The Axisymmetric Case", International Mathematical Forum, Vol. 2, No. 43, pp. $2109-2139,2007$.

[23] Fassò, F., "The Euler-Poinsot Top: A Non-commutatively Integrable System Without Global Action-Angle Coordinates', Z. Angew. Math. Phys. 47, pp. 953-976, 1996.

[24] Bate, R.R., Mueller, D.D. and White, J., "Fundamentals of Astrodynamics”, Dover Books, pp. 13, pp. 156-159, 1971.

[25] Klein F., "The Mathematical Theory of the Top", Chelsea Publishing Company, New York, Lectures delivered in Princeton in 1896

[26] Marsden, J. E. and Ratiu, T.S., "An Introduction to Lie Groups", Introduction to Mechanics and Symmetry, Springer, 2nd Edition, pp. 520, 1999.

[27] Jurdjevic, V., "Geometric Control Theory", Advanced Studies in Mathematics, Cambridge University Press, 52, pp. 169-171, 1997.

[28] Larson, W.J and Wertz, J.R., "Space Mission Analysis and Design", Space Technology Series, 3rd Edition, pp. 366, 1999.

[29] Hughes, P.C., "Spacecraft Attitude Dynamics," Dover Books, pp. 265 1986.

[30] Audin, M., "Spinning Tops: A Course on Integrable Systems", Cambridge Studies in Advanced Mathematics, Cambridge University Press, pp. 52, 1996.

[31] Dubrovin B.A., Krichever I.M. and Novikov S.P., "Integrable Systems I", In: Dynamical Systems IV, Encyclopaedia of Mathematical Sciences, Arnol'd, V.I. and Novikov, S.P., pp. 173-280, Springer, 1990.

[32] Biggs, J. D., "Singularities of Optimal Attitude Motions", 18th IFAC conference on automatic control in aerospace, Nara, Japan, 2010.

[33] Radice, G. and Ali, I., "Autonomous Attitude Control Using Potential Function Method Under Control Input Saturation", In: Proceedings of the 59th International Astronautical Congress, September 2008, Glasgow, Scotland.

[34] Clark, C., McInnes, C.R. and Radice, G., "Space Activities in Glasgow; Advanced Microspacecraft From Scotland", In: Proceedings of the 59th International Astronautical Congress, September 2008, Glasgow, Scotland.

[35] Deb, K., "Optimization for Engineering Design: Algorithms and Examples," PHI Learning Private Limited, pp. 63, 2013. 